This is the final peer-reviewed accepted manuscript of:

Francesco Meoni, Marco Carricato, Optimal selection of the motor-reducer unit in servo-controlled machinery: A continuous approach, Mechatronics, Volume 56, 2018, Pages 132-145

ISSN 0957-4158

The final published version is available online at:

https://doi.org/10.1016/i.mechatronics.2018.11.002

(C) 2018. This manuscript version is made available under the Creative Commons AttributionNonCommercial-NoDerivs (CC BY-NC-ND) 4.0 International License

(http://creativecommons.org/licenses/by-nc-nd/4.0/) 


\title{
Optimal Selection of the Motor-reducer Unit in Servo-controlled Machinery: A continuous Approach
}

\author{
Francesco Meoni $^{\mathrm{a}, *}$, Marco Carricato ${ }^{\mathrm{a}}$ \\ ${ }^{a}$ Dept. of Industrial Engineering, University of Bologna, Viale Risorgimento 2, 40136 \\ Bologna, Italy
}

\begin{abstract}
This papers deals with the optimal selection of motors and reducers in servocontrolled machines. A model of an electromechanical servo-axis is developed, which allows electrical and mechanical losses to be evaluated. The model is based on data that can be extracted from commercial catalogs, thus requiring no experimental characterization. Then, a novel optimal selection procedure is presented, called continuous optimization, based on the extension of a discrete commercial catalog to a continuous one, by means of data fitting on the electromechanical parameters that influence the motor and reducer operation. A non-linear constrained optimization problem is solved in order to find the optimal motor-reducer unit with respect to a variety of objective functions, such as motor size, overall energetic efficiency, total mass, etc.

Keywords: servo-axis, servo-motor, gear reducer, optimal selection, energy efficiency
\end{abstract}

\section{Introduction}

\subsection{State of the art}

A servo-controlled machine, particularly an automatic one, can perform tasks that involve coordinated or synchronized actuation of a significant number of

\footnotetext{
${ }^{*}$ Corresponding author

Email addresses: francesco.meoni2@unibo.it (Francesco Meoni), marco.carricato@unibo.it (Marco Carricato)
}

Preprint submitted to Mechatronics

November 9, 2018 
servo-axes, namely one degree-of-freedom (DoF) electromechanical actuators. Each servo-axis comprises an electric drive with a power electronic converter (e.g. an inverter, exchanging energy with a DC-BUS), a servo-motor (typically, a permanent-magnet synchronous motor, or PMSM), a mechanical transmission and an end-effector.

Since up to dozens or hundreds of servo-axes may be installed in a single servo-controlled machine, the definition of an optimal procedure for component selection is the key to an efficient design. Several issues may arise in this context. First, the available information may be inadequate to accurately account for the actual impact of the components that are chosen, since the model may be incomplete. For instance, a precise dynamic analysis cannot be performed before all components are chosen, and yet components cannot be properly chosen before the dynamic analysis is performed. In particular, the sizing of the motorreducer unit has a great impact on the dynamic performance and the energetic efficiency of the system, and the goal of this work is to define a method that allows the selection of the best motor-reducer pair that minimizes a user-defined objective function.

The optimal selection of servo motors and gear reducers has been studied by several authors in the literature. Pasch and Seering [1] introduced the concept of inertia matching and found the best transmission ratio for inertial loads. Van 25 de Straete et al. 2] proposed a robust procedure for motor selection, introducing a partially automated method that discards motors that are inadmissible regardless of the gear transmission ratio. These works assumed the gearbox to be ideal, i.e. having zero inertia and unit efficiency.

Roos et al. 3] introduced reducer's mechanical efficiency and inertia in the process: these quantities are set as constant during the selection stage, and then verified. Cusimano proposed a method to select the motor via graphical diagrams for an inertial load in [4, and the concept was further extended in [5] 6]. Giberti et al. [7, 8] presented a different approach to obtain similar results: a performance index, called the accelerating factor, was defined for every commercially-available motor; the index was then compared with the load factor 
(representing loading conditions) to verify the selection feasibility. The relationship between the accelerating factor and motor dimensions was investigated in [9], in order to determine top-performance machine.

As for the choice of objective functions, the root-mean-square (RMS) value 40 of the motor torque is normally used as the most representative index for the selection of a servo-motor, since the thermal criterion for motor admissibility requires the RMS torque to be smaller than the rated torque. Minimizing the RMS torque ensures the selection of the smallest possible motor, since the size (and thus the cost) is proportional to the rated torque. Besides, a selective diagram-based procedure based on maximizing the peak torque was proposed in $[10$.

The effect of electric parameters in motor selection was also investigated. Roos et al. 3] introduced power losses due to Joule effect, and used energy efficiency as a criterion to select a motor among several candidates after a firstround mechanical selection. Rezazadeh and Hurst [11 presented an analytical expression for the optimal transmission ratio that increases the energetic efficiency of the motor, considering Joule resistive losses and an ideal gear reducer. Verstraten et al. [12, 13, presented an electromechanical model for a mechatronic application featuring a geared DC motor, showing the electric efficiency 55 map. In [13], different energy computation methods were compared, opening a discussion about the appropriate definition of motor efficiency.

In the aforementioned literature, the selection procedure requires the evaluation of a discrete set of candidate motors and gear reducers. Usually, the problem is simplified by neglecting the reducer real characteristics (inertia and efficiency) in the first stage. Feasibility is verified by taking the non-ideal reducer characteristics into account a posteriori, thus making the process iterative.

\subsection{A novel optimization approach}

In this work, we propose a novel optimization procedure that avoids the iterative process and performs an optimal choice in its most general case. First, ${ }_{65}$ we present an electromechanical model of the servo-axis, which takes electrical 
(energy loss in the drive and the motor) and mechanical quantities (inertias and efficiencies for every component, including the reducer) into account. In particular, the electric model features the introduction of core losses and saturation losses, which influence the motor torque-current relation. A key feature of the presented model is that it is based on parameters that can be inferred from rated data commonly available in commercial catalogs, thus requiring no experimental characterization of components.

Then, the optimal selection problem is addressed by introducing a novel procedure called continuous optimization. The nature of commercially available catalogs is intrinsically discrete, since the available choices are a finite number among the range of components' size. In this paper, we present a method that extends a discrete (commercially available) catalog to a continuous one by fitting manufacturer's data. A size index is introduced to map the range of available components. Every real value of the size index inside the range corresponds to 80 a virtually available choice, with its own set of characteristic parameters. This allows the optimal selection problem to be tackled in the most general case, since the relation between constructive parameters and size is inferred a priori. This also allows the feasibility range for the selection of commercial components to be unambiguously determined.

The best size of motor and reducer may be found with respect to several objective functions. Among others, the pair that maximizes energy efficiency of the global servo-axis may be looked for. The minimization of the energy absorbed by the servo-axis is crucial in a scenario where up to hundreds of servo-axes are simultaneously running in the same machine. In other contexts, other objective functions can be chosen, e.g. the overall mass in aeronautical applications. Once mechanical and electrical parameters in the servo-axis model are expressed as continuous functions of the motor and reducer size indexes, whichever objective function is chosen, the optimization process may be carried out in substantially the same way.

95

It is worth observing that standard commercial components reflect stateof-the-art designs. This implies that similar-size components normally posses 
similar characteristic features, regardless of the manufacturer. For example, the rotor inertia of a PMSM with a given rated torque normally changes little from manufacturer to manufacturer. Minor differences may be present, but it can be conjectured that a continuous catalog depends only to a limited extend on the manufacturer the original data are taken from (especially if these data are approximated, rather than strictly interpolated), thus providing a general tool.

Once an optimal solution is found, several scenarios are possible. It may happen that the optimum is near a commercially-available choice: in this case the continuous optimization procedure gives a strong and accurate indication for a first-try selection. Indeed, each commercial solution is identified as feasible or unfeasible, without needing further verification. Alternatively, the optimum may be reasonably far from available choices. In this case, the optimization highlights a lack of preferred choices in a particular range of the catalog. Once such an 'underpopulated' range is found, several solutions may be available. In an industrial environment, component selection is ordinarily made in a pool of components that is smaller than the full catalog, since this grants several advantages (a faster supply from manufacturers, cost reduction due to stock purchase, reduced storing and management costs, etc.). The optimal solution, however, may this way be overlooked. The knowledge of a theoretical optimum may allow the inclusion of different component sizes in the selection pool. Switching manufacturer is also an alternative, since a different discrete catalog may come with a different resolution, and thus offer different sizes. Finally, custom-designed components may be used in specific applications. When a single machine comprises up to hundreds of servo axes, it may justify the development of tailor-made solutions. The same is true when a single motor-reducer unit has a significant value.

\subsection{Outline}

Section 2 presents the mechanical and electrical model of the servo-axis. All losses along the servo-axis, from the power electronic converter (PEC), to the end-effector, are evaluated, including winding and iron losses. Section 3 
presents the continuous optimization procedure for selecting the moto-reducer unit. Size indexes are defined and a discrete commercial catalog is extended to a continuous one. The resulting non-linear optimization problem is solved via the interior-point algorithm. Section 4 applies the presented approach to two industrial cases: a single servo-axis with a large payload and a multi-axis automatic machine with purely inertial loads.

\section{Modelling}

This Section presents the model of a servo-axis, from the power electronic converter (PEC) connected to the electric grid to the end-effector. In industrial environment, the power layer is normally represented by a DC-BUS, which distributes the power between servo-axes. A typical DC-BUS architecture employs PMSM motors fed by 3-phase alternate tension (also referred as AC brushless). Therefore, each motor is driven by an inverter that acts as a PEC, which is the case considered from now on.

The servo-axis model is divided into two parts: the mechanical part and the electrical part. The former comprises the end-effector, the transmission chain, and the motor rotor. The main mechanical variables are the motor's torque $M$ and rotational speed $\omega_{m}$. The profiles of $M$ and $\omega_{m}$ during the operation cycle define the motor operating range.

The electric model is divided, in its turn, into two parts. The first one is the electric model of the motor, which correlates the mechanical quantities $M$ and $\omega_{m}$ with the electric variables $I$ and $\omega: I$ is the effective current flowing through the PMSM stator, whereas $\omega$ is the excitation frequency of the source.

150 The second part is responsible for evaluating the electric losses in the motor and the inverter, based on the values of $I$ and $\omega$.

\subsection{Mechanical model}

Figure 1 shows the mechanical model of the servo axis, comprising the motor, a gear reducer, and a generic load. The PMSM is modeled as the inertia $J_{m}$ 


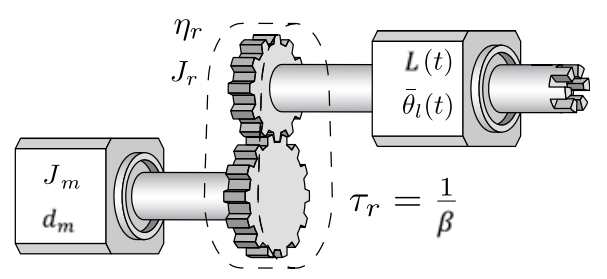

Figure 1: Mechanical model of the servo axis.

$$
\begin{aligned}
M(t) & =\left(J_{m}+J_{r}\right) \dot{\omega}_{m}(t)+\frac{L(t)}{\beta \hat{\eta}_{r}}+d_{m} \omega_{m}(t) \\
& =\beta\left[\left(J_{m}+J_{r}\right) \dot{\omega}_{l}(t)+d_{m} \omega_{l}(t)\right]+\frac{L(t)}{\beta \hat{\eta}_{r}}
\end{aligned}
$$

The efficiency function $\hat{\eta}_{r}$ is defined as:

$$
\hat{\eta}_{r}= \begin{cases}\eta_{r, d i r}, & L \omega_{l}>0 \\ \eta_{r, \text { inv }}^{-1}, & L \omega_{l}<0\end{cases}
$$

where $\eta_{r, d i r}$ and $\eta_{r, i n v}$ are reducer's efficiencies respectively in direct and inverse motion, which are generally different and may depend on the transmitted torque [14]; $L \omega_{l}$ is the power that the load exchanges with the reducer.

The load profile, namely $\omega_{l}(t)$ and $L(t)$, depends on both the task to be performed (i.e. the end-effector motion profile and the external load) and the transmission chain, whose inertias and geometrical features are assumed to be known. The transmission chain is described by a lumped-parameter model, shown in Fig. 2 composed by $N$ blocks, representing a series of one-inputone-output mechanisms. We denote the kinematic state of the end-effector by 


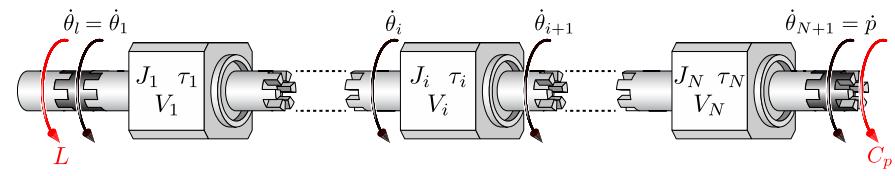

Figure 2: Serial transmission model for a servo-axis with $N$ blocks.

$\bar{p}(t) \triangleq(p, \dot{p}, \ddot{p})^{T}$, and the external load acting on it by $C_{p}$. The $i$ th block connects node $i$ to node $i+1$, and each node has a kinematic state $\bar{\theta}_{i}(t) \triangleq\left(\theta_{i}, \dot{\theta}_{i}, \ddot{\theta}_{i}\right)^{T}$. Each block is characterized by a transmission ratio $\tau_{i}$ (defined as $\dot{\theta}_{i+1} / \dot{\theta}_{i}$ ), an inertia $J_{i}$ reduced to $\theta_{i}$, and a potential energy $V_{i}$. All these quantities are function of position $\theta_{i}$. At the extremities of the chain, we have $\bar{\theta}_{1}=\bar{\theta}_{l}$ and $\bar{\theta}_{N+1}=\bar{p}$. Once the transmission chain and the end-effector motion profile $\bar{p}$ are known, the kinematic state $\bar{\theta}_{l}$ of the reducer output shaft is found by inverse kinematics.

Denoting the torque at the $i$ th node as $C_{i}$, the equilibrium of the $i$ th block yields:

$$
C_{i}=J_{i} \ddot{\theta}_{i}+\frac{1}{2} \frac{d J_{i}}{d \theta_{i}} \dot{\theta}_{i}^{2}+\frac{d V_{i}}{d \theta_{i}}+\frac{\tau_{i}}{\hat{\eta}_{i}} C_{i+1}
$$

where mechanical losses in each block are taken into account by means of global efficiencies ${ }^{1} \eta_{i, d i r}$ and $\eta_{i, i n v}$, respectively valid for direct and inverse power flow, so that:

$$
\hat{\eta}_{i}= \begin{cases}\eta_{i, d i r}, & C_{i+1} \dot{\theta}_{i+1}>0 \\ \eta_{i, i n v}^{-1}, & C_{i+1} \dot{\theta}_{i+1}<0\end{cases}
$$

Starting from the end-effector side, where $C_{i+1}=C_{p}$, Eq. (3) is applied backwards until the load torque $C_{1}=L$ is found.

\footnotetext{
${ }^{1} \mathrm{~A}$ more detailed friction model may be implemented, such as Coulomb friction. However, it would require the computation of reaction forces by means of multibody analysis, making the mechanical model more complicated while real would be uncertain.
} 


\subsection{Electrical model}

185 and the inverter.

\subsubsection{Motor model: Torque-current and frequency-speed relations}

This section introduces the operative model of the motor, linking the motor mechanical parameters (rotational speed $\omega_{m}$ and torque $M$ ) to its electric operating conditions (exciting frequency $\omega$ and stator current $I$ ). In a synchronous machine, there is no slip and the rotor is locked to the rotating magnetic field generated by the stator. Hence, indicating the number of pole pairs by $p$, the rotor speed $\omega_{m}$ is proportional to the frequency $\omega$ of the exciting source, so that $\omega_{m}=p \omega$.

The motor torque $M$ depends on both constructive parameters, such as stator inductances and magnetic-field intensity, and operating conditions, in particular stator currents [15]. Accordingly, $M$ can be expressed as:

$$
M=g(I) \quad \Leftrightarrow \quad I=g^{-1}(M)
$$

where function $g$ depends on constructive parameters. The most common and widely-spread torque-current model is based on a linear proportion, such as $M=k_{T} I$ [16, 17]. Coefficient $k_{T}$, called torque constant, is listed for every motor on manufacturers' catalogs. This assumption is simple, but it does not consider the effect of saturation of materials and the influence of energy losses when solving the motor equivalent circuit. We prefer to define a novel function $g$ that takes losses into account with a reasonable level of details, though using only rated data readily available from manufacturers. In fact, the evaluation of motor internal parameters, such as self and mutual inductances, can be done experimentally, but it is impractical or unfeasible when an entire motor catalog has to be characterized.

Manufacturers' catalogs usually provide the currents absorbed by the motor while it generates the rated torque $M_{n}$, the maximum peak torque $M_{\max }$, and 


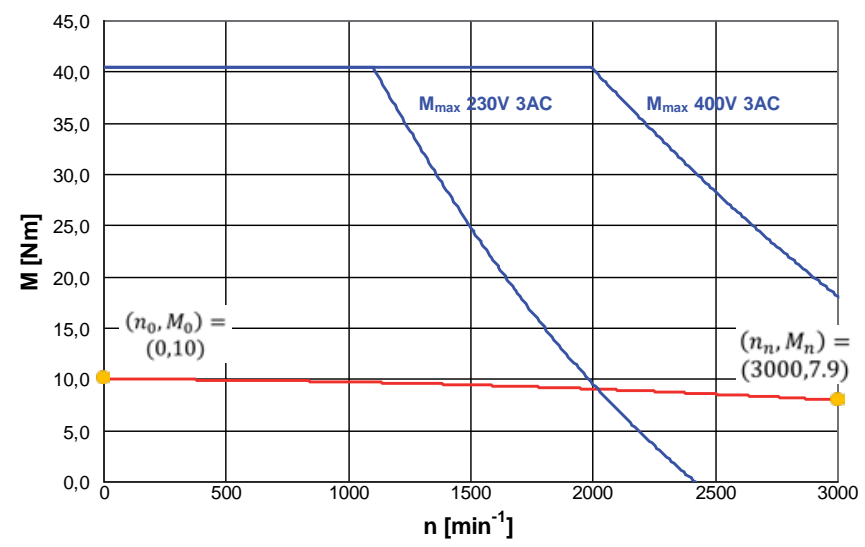

Figure 3: SH100 motor characteristics [16]: $\left(n_{0}, M_{0}\right)=(0,10),\left(\omega_{m, n}, M_{n}\right)=(3000,7.9)$, $n_{\max }=6000 \mathrm{rpm}$.

the stall torque $M_{\mathrm{d}}^{2}, M_{0}$ is usually higher than $M_{n}$, and both values lie on the continuous-service limit curve (see Fig. 3). $M_{\max }$ is the value that the motor can instantaneously provide without damaging conductors; depending on voltage source, $M_{\max }$ may decrease for high rotating speeds. We indicate currents correspondent to $M_{n}, M_{\max }$ and $M_{0}$ as $I_{n}, I_{\max }$ and $I_{0}$, respectively. We propose a model with quadratic torque-current dependence, namely:

$$
M(I)=g_{q}(I)=k_{t 1} I+k_{t 2} I^{2}
$$

which satisfies the constraint $M(0)=0$, since no torque is produced with zero current, and subscript $q$ emphasizes the quadratic relation between $M$ and $I$. Coefficients $k_{t 1}$ and $k_{t 2}$ are determined by imposing:

$$
\left\{\begin{array}{c}
M\left(I_{0}\right)=k_{t 1} I_{0}+k_{t 2} I_{0}^{2}=M_{0} \\
M\left(I_{n}\right)=k_{t 1} I_{n}+k_{t 2} I_{n}^{2}=M_{n} \\
M\left(I_{\max }\right)=k_{t 1} I_{\text {max }}+k_{t 2} I_{\text {max }}^{2}=M_{\text {max }}
\end{array}\right.
$$

\footnotetext{
${ }^{2}$ In practice, the stall torque is measured at a very low non-zero speed, in order to have a uniform distribution of winding temperature. Here, for the sake of simplicity, we assume that $M_{0}$ is measured at $\omega_{m}=0$.
} 
Since system (7) is overdetermined, a least squares fitting method is used to find a pair of values $k_{t 1}, k_{t 2}$ that minimizes the sum of the squares of the residuals.

As long as the current direction flow is irrelevant (such as when evaluating winding losses that depend on square current), current can be evaluated by its magnitude alone. That being said, the torque in Eq. (6) is intended as the magnitude of the actual torque, which can be positive or negative. With this observation, $g$ is a bijective function with domain $I \in\left[0, I_{\max }\right]$ and co-domain $M \in\left[0, M_{\max }\right]$. Accordingly, the inverse function of $g$ for the torque-current conversion is:

$$
I=g_{q}^{-1}(|M|)=-\frac{k_{t 1}}{2 k_{t 2}}+\frac{\sqrt{k_{t 1}^{2}+4 k_{t 2}|M|}}{2 k_{t 2}}
$$

Since the curve in Eq. (6) is generally concave and, thus, $k_{t 2}$ is negative, the solution corresponding to $-\sqrt{k_{t 1}^{2}+4 k_{t 2}|M|}$ occurs after the curve peak and, thus, after the point $\left(I_{\max }, M_{\max }\right)$, so that it can be ruled out.

In Fig. 4 a comparison between the linear and the quadratic torque-current relation is shown for motor SH100/30 by ELAU. It emerges that the linear model can be used to approximate the relation between torque and current until $I$ reaches a certain value beyond which saturation phenomena and iron losses cause a decrease in the ratio of torque versus current (e.g. for B\&R motors, such a value is identified by the manufacturer in twice the nominal current $I_{n}$ [17]). In the figure, the quadratic model is validated by comparison with experimental data.

\subsubsection{Electrical losses}

If $P_{m}$ is the mechanical power to the motor shaft and $P_{e l, t o t}$ is the electric power supplied by the DC-bus to the servo axis, then $P_{e l, t o t}>P_{m}$. The difference between $P_{e l, t o t}$ and $P_{m}$ is due to several sources of dissipation, in both the motor and the inverter. As far as the motor is concerned, two main sources of losses are considered: copper losses $P_{C u}$ (also known as winding losses) and iron losses $P_{F e}$ (also known as core losses).

Copper losses are due to Joule effect and they are proportional to square 


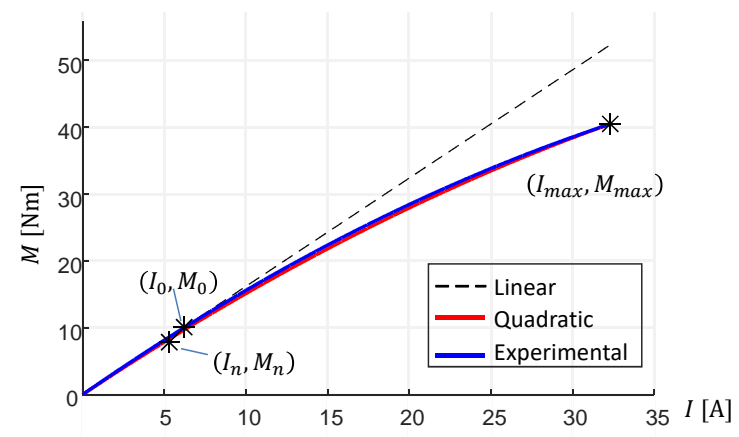

Figure 4: Confrontation of linear, quadratic and experimental models for ELAU motor SH10030 (self-cooled).

current and stator resistance $R$ :

$$
P_{C u}=d_{r} R I^{2}
$$

where $d_{r}$ is a coefficient that depends on how winding resistance is specified 230 by manufacturers (line-to-line, neutral-to-line, etc.). For a sinusoidal threephase PMSM and line-to-line resistance, $I$ is the effective current and $d_{r}=3 / 2$. $R$ is mostly determined by stator conductors' geometry and material, and is ordinarily provided by motor manufacturers' catalog $\AA^{3}$.

Iron losses are caused by time-varying magnetic fluxes in metallic materials, and they are proportional to the fundamental excitation frequency $\omega$. Iron losses can be further divided into two subtypes: hysteresis losses $P_{H y}$ are caused by magnetic hysteresis in the material, whereas eddy-current losses $P_{E d}$ are due to Joule-effect dissipation associated with induced currents.

As for hysteresis losses, in a non-transient operation, the ferromagnetic materials used in the construction of electric machines absorb a quote of energy [18. This energy is proportional to the intensity of the flux density of the mag-

\footnotetext{
${ }^{3}$ In addition to geometry and material, there are various other factors that influence electrical resistance, such as temperature, which in its turn is influenced by the current flowing in the conductors. For the sake of simplicity, this influence is disregarded, and the average constant value provided by manufacturers' catalogs is used here.
} 
netic field, depends on the material itself, and is dissipated by heat. During periodic excitation, every time a cycle is completed, a quote of energy is lost. Due to the energy-per-cycle nature of the dissipation, hysteresis losses $P_{H y}$ are directly proportional to excitation frequency $\omega$. In order to evaluate these losses, empirical equations are usually adopted [19].

The time-varying magnetic flux also causes the induction of an electric field. In magnetic materials, this electric field generates induced currents that are source of additional Joule-effect losses, which are referred as eddy current losses $P_{E d}$. In general, $P_{E d}$ increases as the square of the excitation frequency, and it is proportional to the square of the flux density peak value [20, 21].

Ultimately, both hysteresis losses and eddy currents are proportional to peak flux density and excitation frequency $\omega$. A simplified model is presented in [20] and 22, where an average value is used for flux density. The result shows that the main influence of core losses is the excitation frequency. Accordingly, hysteresis and eddy-current losses are assumed to be proportional to $\omega$ and $\omega^{2}$, respectively:

$$
P_{H y}=k_{f 1}|\omega|, \quad P_{E d}=k_{f 2} \omega^{2}
$$

where frequency $\omega$ can be replaced by rotational speed, since $\omega_{m}=p \omega$.

The overall electric losses $P_{e l, m}$ concerning the motor may thus be expressed as:

$$
P_{e l, m}=P_{C u}+P_{H y}+P_{E d}=d_{r} R I^{2}+k_{f 1}\left|\omega_{m}\right|+k_{f 2} \omega_{m}^{2}
$$

Coefficients $k_{f 1}$ and $k_{f 2}$ are usually not provided by motor manufacturers. A method to infer their values from motor rated data is presented in subsequent Sec. 2.3

As far as the inverter is concerned, the main source of losses is related to switching 23 . When switching between conduction states, there is a transient period where both voltage and current are non-zero. This causes a power loss proportional to switching frequency $f_{s}$. Besides, conduction losses and loadindependent losses are also present.

By including all these factors, the power loss $P_{i n v}$ can be modeled by an 
experimental curve depending on current according to [22]:

$$
P_{i n v}=k_{i 0}+k_{i 1}|I|+k_{i 2} I^{2}
$$

Coefficients $k_{i}$ 's depend on the switching frequency and have to be experimentally determined for several frequency values. Unfortunately, this approach is limited by the scarce availability of inverter experimental data. A fitting of data available for some manufacturer can be done to associate parameters of modules having similar size but produced by different manufacturers, as long as the main characteristics remain the same.

At last, the total electric power drawn by the servo-axis can be calculated by adding mechanical power $P_{m}$ to all electric losses, namely:

$$
\begin{aligned}
P_{e l, t o t}= & P_{m}+P_{C u}+P_{E d}+P_{H y}+P_{i n v}= \\
= & M \omega_{m}+d_{r} R I^{2}+k_{f 1}\left|\omega_{m}\right|+ \\
& +k_{f 2} \omega_{m}^{2}+k_{i 0}+k_{i 1}|I|+k_{i 2} I^{2}
\end{aligned}
$$

By introducing the torque-current model, the electric power is expressed as a function of the motor operation point, i.e. as a function of torque $M$ and speed $\omega_{m}:$

$$
\begin{aligned}
P_{e l, t o t}\left(M, \omega_{m}\right)= & M \omega_{m}+\left(d_{r} R+k_{i 2}\right)\left(g^{-1}(M)\right)^{2}+ \\
& +k_{f 1}\left|\omega_{m}\right|+k_{f 2} \omega_{m}^{2}+k_{i 0}+k_{i 1}\left|g^{-1}(M)\right|
\end{aligned}
$$

\subsection{Determination of coefficients for iron losses}

In order to evaluate the motor electric losses in Eq. (11), the thermal model of the motor should be taken into account. In fact, the heat caused by energy dissipation raises the winding temperature by a quantity $\Delta T$ with respect to (standard) environmental temperature $T_{e n v}$, thus influencing electrical quantities that depend on temperature. The development of a detailed thermal model is not trivial, and goes beyond the scope of this work. However, in order to infer coefficients $k_{f 1}$ and $k_{f 2}$ in Eq. 11] a simplified approach may be adopted.

For thermal equilibrium, in steady-state operations it must be:

$$
P_{e l, m}=\frac{\Delta T}{R_{t h}}
$$



curve provided by the motor manufacturer (see Fig. (4)), then setting $I_{2}=$

\footnotetext{
${ }^{4}$ Here we assume that the motor thermal limit is reached at the motor nominal voltage. In fact, the data that the catalog provides about the stall point refer to this particular condition. This assumption holds for AC brushless [22, but it is not necessarily valid for other types of motor (e.g. DC motors). In this case, an adaption of the model is in order.
} 
$g_{q}^{-1}\left(\left|M_{2}\right|\right)$. Finally, we have:

$$
\left\{\begin{array}{l}
d_{r} R I_{0}{ }^{2}=d_{r} R I_{n}{ }^{2}+k_{f 1}\left|\omega_{m, n}\right|+k_{f 2} \omega_{m, n}^{2} \\
d_{r} R I_{0}{ }^{2}=d_{r} R I_{2}{ }^{2}+k_{f 1}\left|\omega_{m, 2}\right|+k_{f 2} \omega_{m, 2}^{2}
\end{array}\right.
$$

from which $k_{f 1}$ and $k_{f 2}$ are easily computed.

If $\left(M_{2}, \omega_{m, 2}\right)$ cannot be determined with sufficient precision on the continuousservice limit curve, more points can be used, and $k_{f 1}$ and $k_{f 2}$ can be calculated by a least-square fitting method 5 .

Notice that, when $P_{e l, m}^{*}, k_{f 1}$ and $k_{f 2}$ are known, Eq. (16) allows one to compute the stator current in limit continuous-service operation as:

$$
I_{l i m}=\sqrt{\frac{1}{d_{r}} \frac{P_{e l, m}^{*}-k_{f 1}\left|\omega_{m}\right|-k_{f 2} \omega_{m}^{2}}{R}}
$$

and, thus, to obtain an analytical expression of the continuous-service torquespeed limit curve:

$$
M_{l i m}\left(\omega_{m}\right)=g\left(I_{l i m}\right)
$$

For the Elau SH100 motor, represented in Fig. 3. we have: $M_{2}=9 \mathrm{Nm}, \omega_{m, 2}=$ $2000 \mathrm{rpm}, R=1.81 \Omega, P_{e l, m}^{*}=112.6 \mathrm{~W}, k_{f 1}=0.0043 \mathrm{~W} / \mathrm{rpm}$ and $k_{f 2}=$ $3.44 \cdot 10^{-6} \mathrm{~W} / \mathrm{rpm}^{2}$.

\section{Optimal selection of the motor-reducer unit}

A main issue arises during the selection of a motor-reducer unit: the motor and reducer characteristics, which are unknown at the moment of selection, influence the dynamic behavior of the system. If these quantities are neglected or set as try-out values, the solution accuracy must be verified ex-post under real-case conditions. If the solution proves to be unfeasible or sub-optimal, a new selection must be made, thus making the process iterative.

\footnotetext{
${ }^{5}$ Even more simply, a linear relation between $P_{F e}$ and $\omega_{m}$ can be assumed, thus setting $k_{f 2}=0$ and obtaining: $k_{f 1}=d_{r} \frac{R}{\omega_{m, n}}\left(I_{0}^{2}-I_{n}^{2}\right)$ 22].
} 
In order to avoid iterative steps, we present here a selection method that allows all motor and transmission characteristics (including inertias, efficiencies and electrical loss parameters) to be taken into account at the outset. The method is based on the extension of a discrete commercial catalog to a continuous catalog obtained by fitting manufacturers' data. Further advantages of the presented approach are described in the Introduction.

\subsection{Continuous catalog definition}

Given a list of commercially available components, a size index is defined to identify the size of a single component within the given population. Each component is characterized by a list $\bar{\xi}$ of constructive parameters, generally depending on the size of the component itself.

The parameter used as size representative of the motor is the rated torque $M_{n}$. Motors are sorted with respect to $M_{n}$, from the smallest to the largest, and they are numbered progressively by index $j=1, \ldots, N_{m o t}$, where $N_{m o t}$ is the number of available motors. $\alpha \in \mathbb{R}$ is a continuous size index defined as:

$$
\alpha=\frac{M_{n}}{M_{r e f}}
$$

where the reference value $M_{r e f}$ may be arbitrarily chosen. If $M_{r e f}=M_{n, \max }$, $\alpha=1$ identifies the largest motor. However, while comparing different catalogs, $\alpha$ may be larger than one. $\alpha_{j}$ is the size index corresponding to the $j$-th motor in the catalog, denoted by $\mathcal{M}_{j}$.

The extension of a discrete catalog to a continuous one consists in considering each value of $\alpha \in\left[0, \alpha_{\max }\right]$ as a virtually available choice. We denote by $\bar{\xi}_{\alpha}(\alpha)$ the set of constructive parameters that (continuously) depend on the motor size. Both electric and mechanical parameters are included, such as rotor inertia $J_{m}$, winding resistance $R$, electric loss coefficients, etc. Obtaining curves $\bar{\xi}_{\alpha}$ from rated data may be performed by using several methods, and further details are given in Sec 3.1 .2

The same procedure may be applied for the gear reducer. In this case, the size index is chosen to be the gear ratio $\beta$. Gearboxes are sorted from 
the smallest value of $\beta\left(\beta_{\text {min }}\right)$ to the largest one $\left(\beta_{\max }\right) . \mathcal{R}_{k}$ denotes the $k$ th reducer, with $k=1, \ldots, N_{\text {gear }}$, and $N_{\text {gear }}$ being the number of gearboxes available in the catalog. Commercial catalogs show data only for gearboxes operating as reducers (i.e. $\beta>1$ ), since this is the most common case in practice. A multiplication operation mode (i.e. $\beta<1$ ) may be obtained by utilizing the same component with flipped sides ${ }^{6}$ If both reduction and multiplication operation modes are considered to be possible, $\beta_{\min }=1 / \beta_{\max }$.

As far as the inverter is concerned, an issue arises, since inverter parameters, such as loss coefficients in Eq. 12, are not directly dependent on motor or reducer size indexes. A third size index could be defined to map a discrete inverter catalog. However, this would increase the dimension of the problem and, furthermore, it would be a questionable choice, since the selection of motor and inverter are strictly related. Since the inverter, as the motor, has a maximum value of admissible current, denoted by $I_{i n v, \max }$, we prefer to have the generic motor with current limit $I_{m, \max }$ be assigned the inverter with the smallest value of $I_{\text {inv, } \max }$ such that $I_{m, \max } \leq I_{\text {inv, } \max }$. In other words, we select the smallest inverter that does not limit the motor operating range. By using this criterion, an inverter is unambiguously assigned to each motor $\mathcal{M}_{j}\left(j=1, \ldots, N_{m o t}\right)$. Notice that the same inverter may be assigned to multiple motors, but not viceversa. In this way, the inverter parameters are related to the motor size, thus allowing to include them in the set $\bar{\xi}_{\alpha}$.

The union of the function sets $\bar{\xi}_{\alpha}$ and $\bar{\xi}_{\beta}$ forms the multivariate function $\bar{\xi}(\alpha, \beta)=\left(\bar{\xi}_{\alpha}, \bar{\xi}_{\beta}\right)$.

Several important considerations must be made while mapping one or more discrete catalogs into a continuous one. In order to obtain physically plausible curves, only components of the same family should be considered. For example,

\footnotetext{
${ }^{6}$ Reducer catalogs provide the gearbox inertia reflected to the input shaft. Since there is no way to figure out how inertia is actually distributed between the gearbox shafts, it is assumed that all inertia is concentrated on the input shaft. Thus, disregarding efficiency, the inertia reflected to the output shaft is $J_{\text {out }} \simeq J_{\text {in }} \beta^{2}$.
} 
the type of cooling of a PMSM (forced, liquid, natural) plays a determinant role on motor performances. Motor with similar mechanical characteristics, but different cooling, may have different performances, thus invalidating the extension process. The same issue arises when taking into account the winding arrangement: the same motor (with the same inertia and mass) can be equipped with different winding layouts (e.g. series or parallel connection), thus changing electrical performances, such as current absorption. Some manufacturers give for each motor different winding arrangements, which are reflected in different nominal speeds. These issues may be overcome by solving the problem with different continuous catalogs, one for each family or for each motor version (see Sec. 4). Looking at future developments, an alternative solution could be using more than one index to characterize the motor, representing size, cooling, winding adaptation, etc.

\subsubsection{Evaluation of continuous quantities}

Depending on the selected motor and reducer, the torque on the rotor shaft is (see Eq. (11)):

$$
\begin{array}{r}
M(\alpha, \beta, t)=\beta\left[\left(J_{m}(\alpha)+J_{r}(\beta)\right) \dot{\omega}_{l}(t)+\right. \\
\left.d_{m}(\alpha) \omega_{l}(t)\right]+\frac{L(t)}{\beta \hat{\eta}_{r}(\beta)}
\end{array}
$$

where $J_{m}$ and $d_{m}$ are functions of the motor size, whereas $J_{r}$ and $\hat{\eta}_{r}$ are function of the reducer.

The current flowing in the stator windings is obtained by Eq. (8) as

$$
I(M, \alpha)=g_{q, \alpha}^{-1}(|M|)=-\frac{k_{t 1}(\alpha)}{2 k_{t 2}(\alpha)}+\frac{\sqrt{k_{t 1}^{2}(\alpha)+4 k_{t 2}(\alpha)|M|}}{2 k_{t 2}(\alpha)}
$$

where $k_{t 1}$ and $k_{t 2}$ are motor parameters. Function $g_{q, \alpha}$ has the same properties as the one in Eq. (6), thus it is invertible in the domain $\left[0, I_{\max }(\alpha)\right]$.

Finally, the instantaneous power absorbed by the servo axis is (see Eq. 14p):

$$
\begin{gathered}
P_{e l, t o t}(\alpha, \beta, t)=M \beta \omega_{l}+d_{r}\left(R+k_{i 2}\right)\left[g_{q, \alpha}^{-1}(M)\right]^{2}+ \\
+\beta k_{f 1}\left|\omega_{l}\right|+\beta^{2} k_{f 2} \omega_{l}^{2}+k_{i 0}+k_{i 1}\left|g_{q, \alpha}^{-1}(M)\right|
\end{gathered}
$$




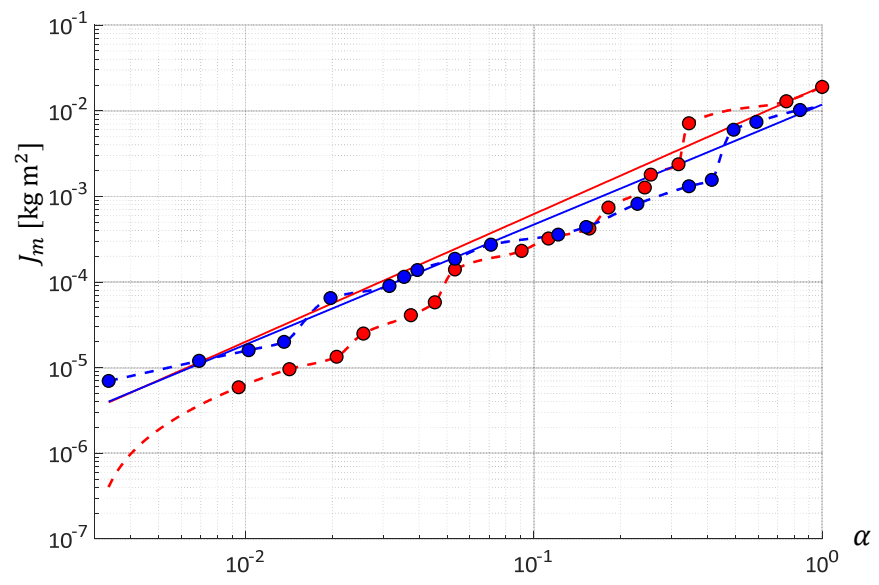

Figure 5: Data fitting for ELAU (red) and B\&R (blue) motor inertias: original data (circles), approximation by power laws (solid lines), and cubic interpolation (dotted lines). (For interpretation of the references to colour in this figure legend, the reader is referred to the web version of this article.)

where coefficients $R, k_{i 1}, k_{i 2}, k_{f 1}, k_{f 2}$ depend on $\alpha, \omega_{l}$ depends on time, and $M$ is reported in Eq. (22).

\subsubsection{Data fitting methods}

The set of functions $\bar{\xi}(\alpha, \beta)$ is obtained by fitting discrete data sets from commercial catalogs; this can ordinarily be done via either interpolation or approximation. The former allows a piece-wise function between data points to be constructed, and it strictly contains original data; in the latter, a generic function is adopted to approximate the original data by using the ordinary least-square regression.

Figure 5 shows an example of data fitting for two different manufacturers relative to the motor inertia $J_{m}$, with respect to motor size $\alpha$, in double logarithmic scale. The red color refers to ELAU motors (SH series [16], reported in Tab. 6), whereas the blue color refers to B\&R actuators (8SL series [17], $\omega_{m, n}=3000$, reported in Tab. 7). The original discrete data are identified by

circles. The solid lines represent approximating functions, which adopt power 
law such $c_{1} \alpha^{c_{2}}$, whose coefficients are determined by least square fitting. The dotted lines denote interpolation by cubic Hermite spline interpolation (cspline). The approximating functions are similar for the two manufacturers, but the difference from the original data are significant, in particular for ELAU motor inertias: for smaller motors the approximate inertia is up to five times the original. On the other hand, the interpolation gives a more accurate representation while being more locally dependent on original data, and thus on the manufacturer.

In general, a continuous catalog obtained by approximation is supposed to be less sensitive to variations of the original data than a catalog obtained by interpolation, thus being expected to depend to a smaller extent on the specific manufacturer data used to generate it. This should ease component selection from different manufactures' catalogs, based on the theoretical optimum. However, since an approximated catalog more loosely adheres to a specific discrete one, it may increase the chance that a theoretical optimum may not have a feasible commercial counterpart. A continuous catalog obtained by data interpolation is expected to have opposite advantages and drawbacks.

A further consideration is in order. The servo-axis parameters cannot assume arbitrary values, since physical constraints must be respected. In some cases, such constraints are explicit and simple to be implemented (e.g. inertia $J_{m}$ or resistance $R$ cannot be negative), but in other cases not they are not. In fact, physical parameters are inherently related, and the combination of them must be consistent: the constraints of one parameter may depend on the value assumed by another one. For example, the numerator inside square root of Eq. 19 must be positive: in fact, this adds a constraint between the values of $R$ and $I_{0}$, contained in the term $P_{e l, m}^{*}$, and the coefficients $k_{f 1}$ and $k_{f 2}$ inferred applying the model.

Therefore, the selection of an approximating function is a delicate and timeconsuming procedure, which may require a dedicated tuning in order to keep ${ }_{420}$ physical parameters inside feasible windows. For example, it may be needed to introduce different functions to cover different ranges of $\alpha$, or adapt them 
depending on the motor type and manufacturer. Alternatively, constrained optimization methods may be utilized while determining the approximating function, to make it compliant with physical constraints.

On the other hand, interpolation grants that the parameters of the "intermediate" motors float in a relatively limited range, thus greatly improving physical consistency. For all these reasons, in the current implementation of our algorithm a strict interpolation was used. This provided reliability to the model and allows for the process to be automatized: in fact, once the interpolation algorithm is chosen, no other adjustments are required. In all case we analyzed, results were physically consistent. As a result, however, the shape of the objective function appreciably depends on the specific catalog database that is used. A thorough analysis of the effects of data fitting on the continuous optimization approach will be the subject of future research.

\subsection{The optimization problem}

The optimization problem consists in the minimization of a user-defined objective function, which depends on both system parameters and operating conditions. In our case, the motor-reducer selection may be considered as a nonlinear programming problem of a generic objective function $f(\alpha, \beta)$ of two unknown variables $\alpha$ and $\beta$. The optimal solution is denoted by $\left(\alpha^{*}, \beta^{*}\right)$. The problem is bounded by limitations introduced by the motor and the gearbox. These limitations are both mechanical (e.g. maximum speed of rotating components) and electrical (e.g. the RMS value of motor torque cannot exceed the continuous service limit). The feasibility constraints are expressed as inequalities, where a given parameter (generally depending on $\alpha, \beta$, and possibly time t) must be smaller than a given bound (usually depending on $\alpha, \beta$, or both).

\subsubsection{Constraints}

The RMS value of the motor torque over a cycle is defined as:

$$
M_{R M S}(\alpha, \beta)=\sqrt{\frac{1}{t_{c}} \int_{0}^{t_{c}} M^{2}(t) d t}
$$


where $t_{c}$ is the duration of the task cycle.

For periodic tasks, the RMS torque value is normally used for the verification of the motor thermal condition. In order to guarantee that the motor does not reach an excessive temperature, the value $M_{R M S}$ must be smaller than the continuous limit $M_{\text {lim }}$. In order to take into account the dependence of $M_{\text {lim }}$ on motor speed $\omega_{m}$ (see Eq. 201), the RMS speed value $\omega_{m, R M S}$ is ordinarily computed, thus checking that the equivalent operating point $\left(M_{R M S}, \omega_{m, R M S}\right)$ ${ }_{455}$ is inside the continuous service zone. Considering the influence of motor speed on $M_{l i m}$ implicitly grants that core losses are also taken into account into thermal verification 7 . Notice that $M_{l i m}$ is a function of both $\alpha$, due to motor size, and $\beta$, since $\omega_{m, R M S}=\beta \cdot \omega_{l, R M S}$

Other constraints on the motor are the maximal instantaneous values for torque and speed. The former is related to limits on both maximum current and voltage, whereas the latter is a mechanical limit related to centrifugal forces on rotating bodies. The reducer may introduce similar additional limitations. Altogether, the motor constraints can be expressed as:

$$
\left\{\begin{array}{l}
M_{R M S}(\alpha, \beta) \leq M_{l i m}(\alpha, \beta) \\
\max (M) \leq M_{\max }(\alpha, \beta) \\
\max \left(\omega_{m}\right)=\beta \max \left(\omega_{l}\right) \leq \omega_{m, \max }(\alpha, \beta) \\
0 \leq \alpha \leq \alpha_{\max } \\
\beta_{\text {min }} \leq \beta \leq \beta_{\text {max }}
\end{array}\right.
$$

where $M_{\max }$ and $\omega_{m, \max }$ are, respectively, the maximum torque and the maxi-

\footnotetext{
${ }^{7}$ The $I^{2} t$ method could be used as an alternative criterion to RMS torque, albeit used for on-line motor protection rather than motor selection. In this paper, the analytical form of the continuous service curve is inferred by catalog data, also taking into account iron losses varying with motor speed. Thus, the constraint on RMS motor torque is to be preferred over the $I^{2} t$ method since it allows a direct confrontation with the continuous service curve and the inclusion of iron losses. On the other hand, the $I^{2} t$ would require an information storable energy, or at least on the intensity and duration of the allowable current peak, which is usually not directly provided in commercial catalogs.
} 
BUS layout, the value of the nominal bus voltage $V_{D C}$ is designed depending on the grid source tension and on the type of converter. For inverters driving AC brushless, the DC-BUS is designed such as the minimum DC tension matches the voltage peak of the $\mathrm{AC}$ source [24].

As for the voltage role on motor selection, manufacturers provide motor characteristic curves for standard values of the source grid tension $(230 \mathrm{~V}$ or 400V [16], 17]). Since this is known for a give application, this automatically implies the correct motor dataset complying with the corresponding DC-BUS tension (see Sec 4.2). Accordingly, the motor nominal voltage is not used as a parameter to choose and it is not required for our electromechanical model.

\subsubsection{Objective functions}

The definition of a suitable objective function depends on specific needs and is therefore strongly task-oriented. For example, if fixed costs are taken into account, the minimization of the motor RMS torque is a natural choice, since the size, and thus the cost, of a PMSM is proportional to the rated torque $M_{n}$.

However, in new-generation machines, several energy fluxes may be present between the DC bus and the servo-axes, and electrical losses have an appreciable impact on global efficiency. In this scenario, the energy drawn by the servo-axis over a period $t_{c}$ may also be a relevant function to minimize. The latter is obtained by integrating the electric power expressed in Eq. 24 over a cycle, namely:

$$
E_{e l, t o t}(\alpha, \beta)=\int_{0}^{t_{c}} P_{e l, t o t}(\alpha, \beta, t) d t
$$

Equation 27) is the most general expression for the energy exchanged by the 
servo axis and it allows one to evaluate the running costs of servo-actuation. As mentioned in Sec. 2.2.2 the evaluation of inverter losses (see Eq. (12) is dependent on the availability of experimental data. If the latter are not available, only the energy absorbed by the motor may be considered, thus neglecting $P_{i n v}$ in $P_{\text {el,tot }}$.

Other continuous objective functions of $\alpha$ and $\beta$ may easily be conceived and considered in the current framework, with no increase of complexity. For instance, in applications where dimensions and weight are especially important, one may wish to minimize the overall mass:

$$
m_{t o t}(\alpha, \beta)=m_{\text {inv }}(\alpha)+m_{\text {mot }}(\alpha)+m_{\text {gear }}(\beta)
$$

Several algorithm are available to solve a non-linear constrained optimization problem [25]. A comparison of these algorithms aimed at determining the most efficient way to solve the problem is out of the scope of this paper. In this work, we used the interior-point algorithm, which follows a barrier approach and implements sequential quadratic programming [26]. The algorithm, which also implements trust regions, shows robustness on several iterations, but no claim that this choice is preferable to others is made here. Since the solver is local, the output depends on the starting point. In order to find the global optimum, a multi start approach is adopted, i.e. the local solver is started from a set of $n_{p}$ points in the function domain. This also allows parallel computing to be effectively used: the higher $n_{p}$, the higher the chance to converge on the global solution. The selection of the best set of starting points is an interesting topic: here, a uniform distribution of starting points on the domain is set, filtering out points that are too close to an already discovered local minimum [27], and refining not-strictly convergent outputs from the local solver by a direct search algorithm 8 [28].

\footnotetext{
${ }^{8}$ The computation time has an order of magnitude of ten seconds. Since the determination of the best numerical method is not the focus of this paper, a comparison between the computation times of different algorithms was not performed.
} 


\section{Case Studies}

515 different fields of application; namely, a single axis with a large payload and a large cycle duration and a multi-axis automatic machine, characterized by periodic inertial loads and high-frequency tasks.

\subsection{Electric winch for sonar positioning}

520 consists in actuating an electric winch mounted on helicopter, used for positioning a sonar. The duty cycle consists of a first phase where the sonar is dropped into the sea: after a displacement in the air, the sonar travels underwater to reach a determined depth, necessary for making measurements. Then a second

${ }_{525}$ phase starts, where the sonar is lifted back to the helicopter. The typical winch design involves a hydraulic motor. However, an auxiliary electric motor must be able to step in in case of a failure of the hydraulic circuit. The auxiliary servo-axis is the focus of our analysis. Other than guaranteeing the payload (i.e. the sonar) to be moved with high accelerations, the crucial goal for the 530

\subsubsection{Load characteristics}

The system is mechanically modeled by a winch of radius $r$, a steel wire and a body of weight $m_{\text {sonar }}$ representing the sonar. The sonar is assumed to translate along the vertical direction, with its kinematic state being identified by $\bar{p}$. The motion law is a piece-wise function consisting of constant-acceleration portions. In both the dropping and the lifting phase, the sonar moves at a reduced speed $\dot{p}_{1}=1.5 \mathrm{~m} / \mathrm{s}$ in the air. Underwater, the dropping speed is $\dot{p}_{2 d}=3.9 \mathrm{~m} / \mathrm{s}$, whereas the lifting speed is $\dot{p}_{2 l}=4.5 \mathrm{~m} / \mathrm{s}$. The acceleration is fixed at $\ddot{p}_{0}=1$ $\mathrm{m} / \mathrm{s}^{2}$, for a total duty cycle of $t_{c}=141.32 \mathrm{~s}$. The helicopter is supposed to 540 operate at $20 \mathrm{~m}$ above sea level, while the sonar reaches an underwater depth of $224 \mathrm{~m}$. The upper part of Fig. 7 shows the velocity $\omega_{l}(t)$ and the acceleration $\dot{\omega}_{l}(t)$ of the winch shaft. 


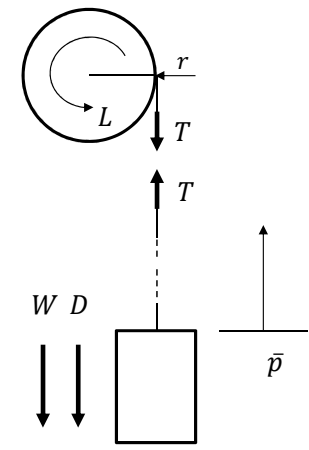

Figure 6: Forces acting on the sonar positioning system: drag force $D$, weight $W$, cable tension $T$, and load torque $L$.

Other than inertia, the forces acting on the sonar are weight $W$ (which includes underwater hydrostatic thrust), cable force $T$ (designed to be always positive), and drag force $D$. The latter is generated when a body moves inside a fluid, and it is proportional to square value of the velocity, other than fluid density, body shape and contact surface. The force equilibrium on the sonar leads to (Fig. 6):

$$
W(t)+D(t)-T(t)+\left(m_{\text {sonar }}+m_{\text {wire }}\right) \ddot{p}=0
$$

where:

$$
\begin{aligned}
& W(t)=g\left[V_{a}(t) \rho_{\text {sonar }}+V_{w}(t)\left(\rho_{\text {sonar }}-\rho_{\text {water }}\right)\right] \\
& D(t)=\frac{1}{2} \rho_{\text {water }} \dot{p}^{2}(t) c_{\text {sonar }} A_{\text {sonar }}
\end{aligned}
$$

$m_{\text {wire }}$ is the mass of the wire portion comprised between the winch and the 550 sonar, $\rho_{\text {water }}$ and $\rho_{\text {sonar }}$ are, respectively, water and sonar density, $g$ is gravity acceleration, $V_{w}$ and $V_{a}$ are the portion of sonar volumes respectively under water and in the air (such as the total volume is $V_{\text {sonar }}=V_{w}+V_{a}$ ), $c_{\text {sonar }}$ is a shape coefficient, and $A_{\text {sonar }}$ is the contact surface perpendicular to the motion direction (Tab. 11). If $J_{\text {winch }}$ is the mass moment of inertia of the winch, the 
load at the winch shaft is thus:

Table 1: Parameters of the sonar positioning system.

\begin{tabular}{|c|c|c|c|}
\hline Parameter & Value & Parameter & Value \\
\hline$J_{\text {winch }}$ & $0.13 \mathrm{kgm}^{2}$ & $\rho_{\text {water }}$ & $1030 \mathrm{~kg} / \mathrm{m}^{3}$ \\
\hline$r$ & $0.15 \mathrm{~m}$ & $\rho_{\text {sonar }}$ & $7850 \mathrm{~kg} / \mathrm{m}^{3}$ \\
\hline$m_{\text {sonar }}$ & $152.4 \mathrm{~kg}$ & $A_{\text {sonar }}$ & $0.13 \mathrm{~m}^{3}$ \\
\hline$m_{\text {wire }}$ & $16.5 \mathrm{~kg}$ & $V_{\text {sonar }}$ & $0.019 \mathrm{~m}^{3}$ \\
\hline$\eta_{\text {winch }}$ & 0.95 & $c_{\text {sonar }}$ & 0.96 \\
\hline
\end{tabular}

The efficiency $\hat{\eta}_{\text {winch }}$ is defined as in Eq. (4). The lower part of Fig. 7 shows the load torque $L(t)$ as it emerges from the mechanical model in Eq. 31.

\subsubsection{Optimization}

The servo-axis is composed by an inverter connected to a DC source (in this case a battery), a forced ventilated PMSM and a parallel-axis gearbox reducer. In particular, Phase motors (U3F series, Tab.4] 29]) and Lenze 2-stages reducers (GFL-06 series, Tab. 8 [30]) are considered. Inverter modules are from B\&R, (ACOPOSmulti series, Tab. 10 [31]) .

From the motor database in Tab. 4 two continuous catalogs are created, according to two different winding arrangements; one with nominal speed $\omega_{m, n}=$ $2000 \mathrm{rpm}$, and one with nominal speed $\omega_{m, n}=3000 \mathrm{rpm}$. For each arrangement, the catalog value of $k_{T}$ is shown as well. The electrical model defined in Sec. 2.2 is applied to each motor in the catalog and the corresponding $k_{t 1}, k_{t 2}, k_{f 1}$ are computed. The complete set of motors data, including the coefficients derived from the electric model, are available at [32]. For this particular motor series, we found that the quadratic term in the model of iron losses (see Eq. (10)) is very small, so that the coefficient $k_{f 2}$ can be approximated to zero with a 

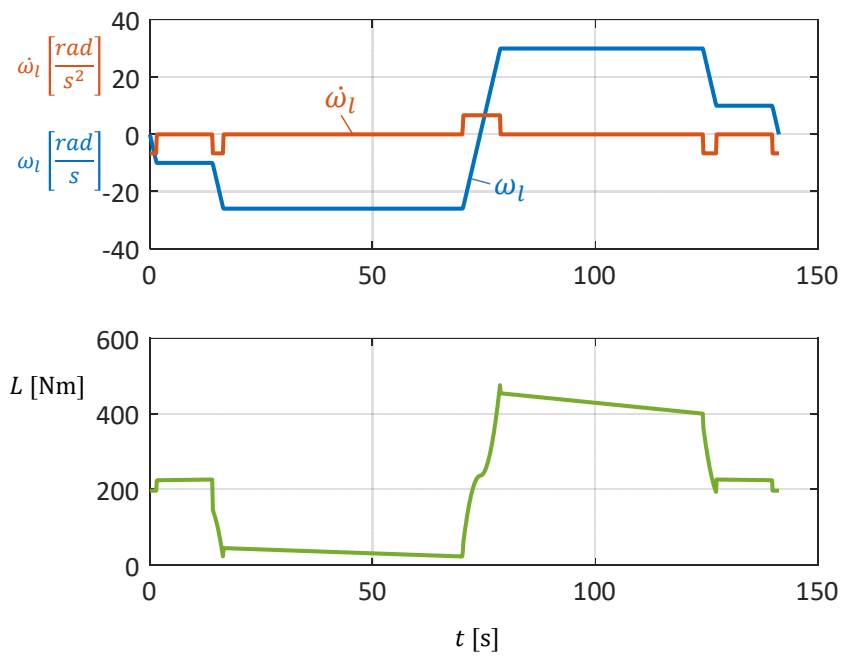

Figure 7: Load profile of the sonar positioning mechanism: velocity $\omega_{l}$ and acceleration $\dot{\omega}_{l}$ in the upper part; load torque $L(t)$ in the lower part.

reasonable accuracy. The coefficient $d_{m}$ is set to zero, since the corresponding damping term in the motor model (see Eq. (1)) is negligible with respect to the drag force term $D$, which is also proportional to velocity.

The set of functions $\bar{\xi}(\alpha, \beta)$ is obtained by fitting the discrete data of the catalog, and in Tab. 2 the list of parameters and the correspondent fitting methods are reported.

The continuous optimization procedure is applied to the winch axis. The implemented constraints are the same as those in Eq. 26). Two objective functions are considered: the main objective function is the servo-axis total mass, but the total energy spent to achieve the task is also important, because minimizing it leads to a smaller (and thus lighter) battery.

Figure 8 shows the results of optimization for the winding arrangement corresponding to $\omega_{m, n}=2000$. For the sake of clarity, the equivalent rated torque $M_{n}$ is represented in the $x$-axis instead of $\alpha$, as in Eq. 21, whereas transmission ratio $\beta$ is on the $y$-axis. The black continuous line represent the feasibility region, where all inequalities are fulfilled. The region coincides with the one de- 
Table 2: Fitting methods for functions $\xi_{i}$. Strict interpolation is used for all parameters.

\begin{tabular}{|c|c|c|c|c|c|}
\hline$\xi_{i}$ & Fitting & Set & $\xi_{i}$ & Fitting & Set \\
\hline$M_{n}$ & linear & $\alpha$ & $R$ & cspline & $\alpha$ \\
\hline$J_{m}$ & cspline & $\alpha$ & $M_{\text {max }}$ & cspline & $\alpha$ \\
\hline$k_{t 1}$ & cspline & $\alpha$ & $k_{t 2}$ & cspline & $\alpha$ \\
\hline$k_{f 1}$ & cspline & $\alpha$ & $k_{f 2}$ & cspline & $\alpha$ \\
\hline$I_{\text {max }}$ & cspline & $\alpha$ & $\omega_{m, \max }$ & linear & $\alpha$ \\
\hline$m_{\text {mot }}$ & linear & $\alpha$ & $\omega_{m, n}$ & linear & $\alpha$ \\
\hline$m_{r e d u c e r}$ & linear & $\beta$ & $k_{i}$ & linear & $\alpha$ \\
\hline$J_{r}$ & linear & $\beta$ & $\eta_{r}$ & linear & $\beta$ \\
\hline
\end{tabular}

fined by the inequality $M_{R M S}(\alpha, \beta)<M_{\text {lim }}(\alpha, \beta)$, which in this case represents

the strictest constraint, and therefore it contains the others. The horizontal black line represents the limit value $\beta_{\text {lim }}$ for which the RMS value of motor speed $\omega_{m, R M S}$ exceeds the rated nominal speed $\omega_{m, n}=2000 \mathrm{rpm}$ (in this case $\left.\beta_{l i m}=\omega_{m, n} / \omega_{l, R M S}=8.58\right)$. The total energy $E_{e l, t o t}$ needed to achieve the task is represented by a color map. The green dots represent the available commercial pairings. The theoretical global energy optimum (red diamond) is $E_{e l, t o t}^{*}=657.7 \mathrm{~kJ}$, found for $M_{n}^{*}=246.5 \mathrm{Nm}$ and $\beta^{*}=7.42$. As for the global mass (see Eq. (28)), for the selected reducer type, the mass $m_{\text {gear }}$ remains constant as the transmission ratio varies. On the other hand, both $m_{i n v}$ and $m_{m o t}$ are monotonically increasing functions of $\alpha$ : thus, the problem of minimizing $m_{\text {tot }}$ reduces to minimizing $\alpha$. In this case, a possible commercial solution is motor $\mathcal{M} 2\left(M_{n, 2}=42.5 \mathrm{Nm}, m_{m o t}=25 \mathrm{~kg}\right)$ with reducer $\mathcal{R} 6\left(\beta_{6}=8.4\right)$, resulting in $E_{e l, t o t}=676.2 \mathrm{~kJ}$. If the most efficient commercial pair were chosen, namely $\mathcal{M} 7\left(M_{n, 7}=260 \mathrm{Nm}, m_{m o t}=138 \mathrm{~kg}\right)$ and $\mathcal{R} 6\left(\beta_{6}=8.4\right)$, then $E_{\text {el,tot }}=658.5$ $\mathrm{kJ}$.

It is worth emphasizing that the feasibility region in Fig. 8 covers the most general case, since all constraints are included. If additional requisites were 


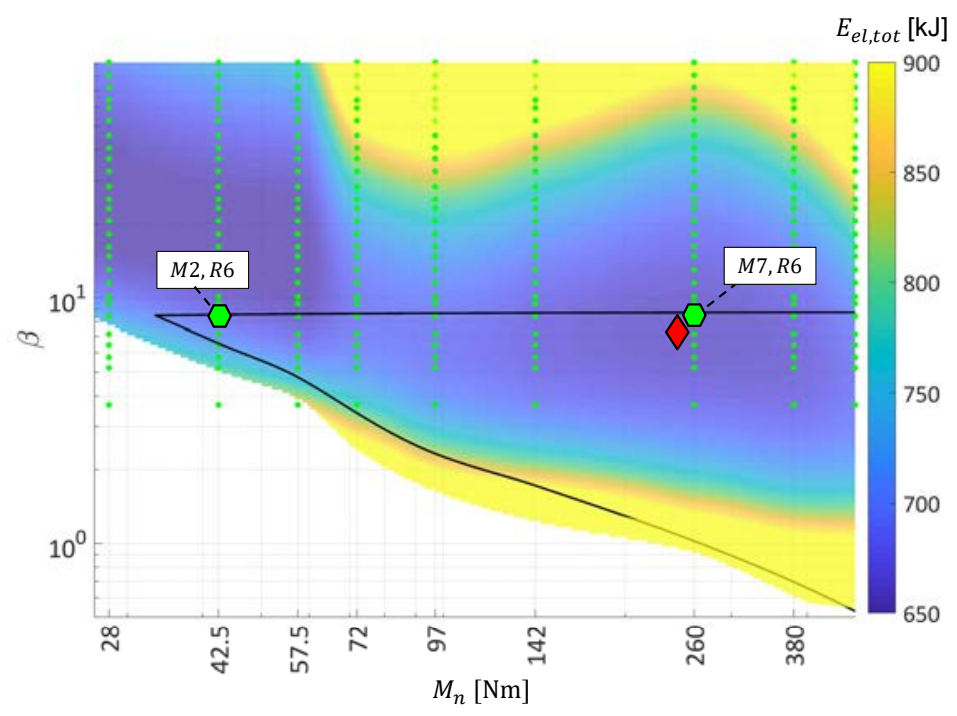

Figure 8: Optimization of the electric winch for Phase motors with $\omega_{m, n}=2000 \mathrm{rpm}$. The black continuous line represent the feasibility region. The theoretical global energy optimum (red diamond) is $E_{e l, t o t}=657.7 \mathrm{~kJ}$, found for $M_{n}^{*}=246.55 \mathrm{Nm}, \beta^{*}=7.42$. The green dots represent the available commercial pairings, while the green hexagons highlight the most convenient commercial solutions, minimizing respectively $m_{t o t}$ (left) and $E_{e l, t o t}$ (right). (For interpretation of the references to colour in this figure legend, the reader is referred to the web version of this article.)

needed, these could be added to the optimization problem in Eq. (26), and very simply dealt with. This implies that all commercial components inside the zone denoted by the black line in Fig. 8 represent feasible solutions, without further verification being needed, at least if strict interpolation were used to fit original catalog data.

Figure 8 gives the opportunity to assesses the wealth of information provided by the continuous approach with respect to the discrete one. A global picture on the trend of the objective function is given. The low energy-consumption zones 615 are easily identified, and it is possible to notice that there is a low-energy zone in the upper left region of the plot which is ruled out by the speed constraint, but that could be effectively explored by using a different catalog. For this reasons, 


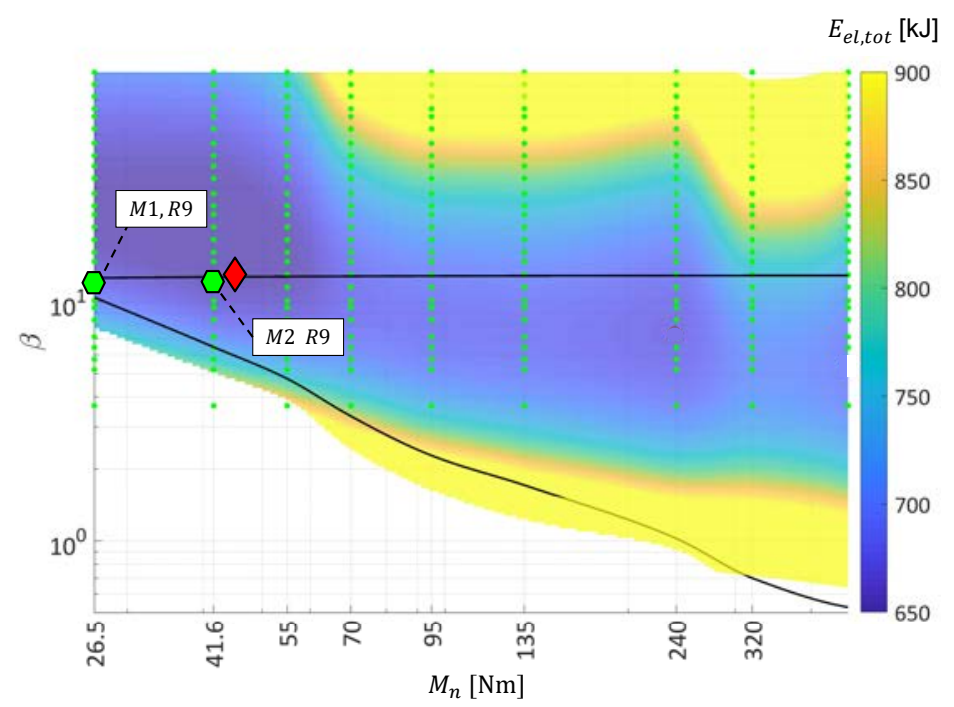

Figure 9: Optimization of the electric winch for Phase motors, with $\omega_{m, n}=3000 \mathrm{rpm}$. The black continuous line represent the feasibility region. The minimum for $E_{e l, t o t}$ (red diamond) is at $M_{n}^{*}=45.14 \mathrm{Nm}, \beta^{*}=12.87$ and $E_{e l, t o t}=648.19 \mathrm{~kJ}$. The green dots represent the available commercial pairings, while the green hexagons highlight the most convenient commercial solutions, minimizing respectively $m_{m o t}$ (left) and $E_{e l, t o t}$ (right). (For interpretation of the references to colour in this figure legend, the reader is referred to the web version of this article.)

the optimization is also run implementing a different winding arrangement of Phase motors, corresponding to nominal speed $\omega_{m, n}=3000 \mathrm{rpm}$ (Tab. 4). The results are shown in Fig. 9 The feasibility zone is wider and the maximum reducer ratio has increased $\left(\beta_{l i m}=\omega_{m, n} / \omega_{l, R M S}=12.87\right)$. The global minimum for $E_{e l, t o t}$ (red diamond) lies on the edge of the region: at $M_{n}^{*}=45.14 \mathrm{Nm}$ and $\beta^{*}=12.87$, it is $E_{e l, t o t}^{*}=648.19 \mathrm{~kJ}$. This catalog better complies with the application requirements: in fact, choosing the closest commercial components to the energy optimum leads to a smaller motor with respect to the previous case, albeit the energy consumption is roughly the same; namely, motor $\mathcal{M} 2$ ( $\left.M_{n, 2}=41.6 \mathrm{Nm}, m_{m o t}=25\right)$ and reducer $\mathcal{R} 9\left(\beta_{9}=11.52\right)$ for $E_{e l, t o t}=654 \mathrm{~kJ}$.

Figure 9 confirms that the most efficient solutions are obtained with small 


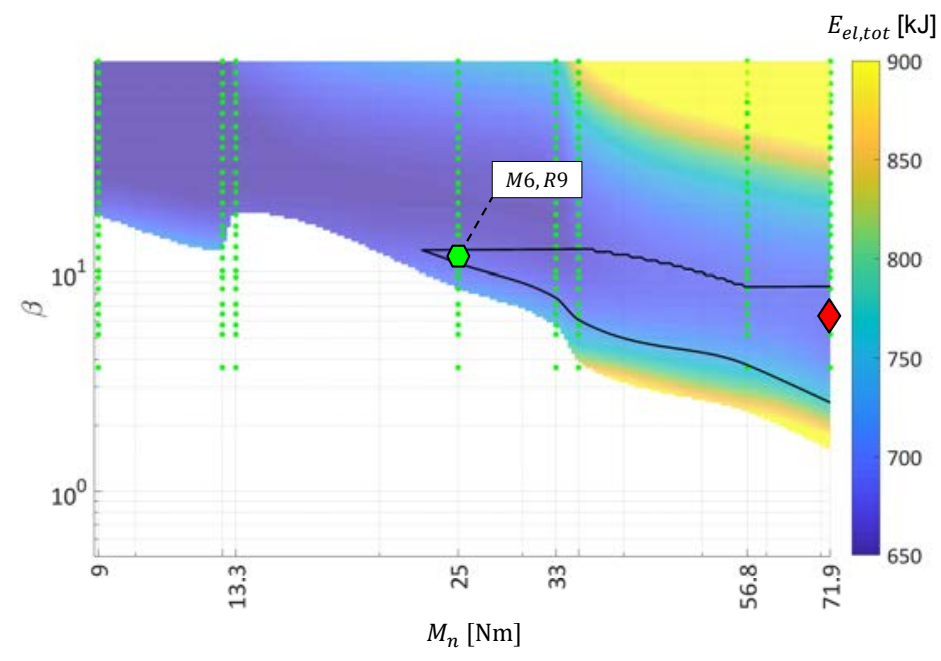

Figure 10: Optimization of the electric winch for ELAU motors. The black continuous line represent the feasibility region. The green dots represent the available commercial pairings, while the green hexagon identifies the commercial solution that minimizes $m_{t o t}$. The energy minimum lies on the boundary. (For interpretation of the references to colour in this figure legend, the reader is referred to the web version of this article.)

motors operating at high velocities (upper-left zone of the graph) and that, in this high-efficiency region, the energy consumption changes little, indeed. Accordingly, the smallest available motor in the catalog may be selected, namely $\mathcal{M} 1$ (with $M_{n, 1}=26.5 \mathrm{Nm}$ and $\left.m_{m o t}=19 \mathrm{~kg}\right)$, paired with reducer $\mathcal{R} 9\left(\beta_{9}=\right.$ 11.52), with an appreciable size and mass reduction, but still a similar energy consumption, i.e. $E_{e l, t o t}=679.8 \mathrm{~kJ}$.

The global trend offered by the continuous optimization approach provides useful indications that allow us to further optimize the selection process. We notice that the feasibility region is expected to continue for smaller values of $\alpha$, i.e. smaller motors than $\mathcal{M} 1$. Accordingly, in order to explore the possibility to mount a smaller motor, it is interesting to analyze the problem implementing a catalog from a different manufacturer (ELAU forced-ventilated series, Tab. 5 [16]), which provides smaller motors. Figure 10 shows the results. Since the catalog contains different rated-speed motors, the reducer-ratio limit is variable 
(no different windings arrangements are available in this case). As expected, the feasibility region continues for smaller motors. The smallest commercially available pairing is motor $\mathcal{M} 6\left(M_{n, 6}=25 \mathrm{Nm}, m_{m o t}=16.6 \mathrm{~kg}\right)$ coupled to reducer $\mathcal{R} 9$. Even though the difference in terms of rated torque is not large, the downsizing in terms of mass is not negligible. The energy consumption in this case is $E_{e l, t o t}=675.72 \mathrm{~kJ}$, which is only $3.2 \%$ larger than the energy consumption obtained with the most efficient solution obtained so far (motor $\mathcal{M} 2$ and reducer $\mathcal{R} 9$ in Fig 9 , yielding $E_{\text {el,tot }}=654 \mathrm{~kJ}$ ).

\subsection{Multi-axes packaging machine}

This application consists in a multi-axis servo-controlled automatic machine for packaging designed by IMA S.p.A. The production line employs up to hundreds of servo motors, but we focus our analysis on the section responsible for folding a die-cut blank to obtain a box. This section contains 8 different servoaxes, used to drive several linkages. In this scenario, the reduction of energy consumption is highly impactful, given the number of servo-axes to be optimized. Other than that, additional selections constraints may come from logistic reasons (e.g. the necessity to reduce the number of different components) and mechanical design choices (e.g. the inclusion of no gearbox reducers to limit clearances and improve reliability).

\subsubsection{Load characteristics}

The machine operates with a productivity of 450 beat-per-minute, which corresponds to a cycle time of $t_{c}=0.133 \mathrm{~s}$. Each axis actuates a linkage and performs a periodic motion. Table 3 shows a summary of the load characteristics for each axis, denoted $\mathcal{A} 1$ through $\mathcal{A} 8$, with the type of the actuated mechanism. Notable axes are $\mathcal{A} 5$ and $\mathcal{A} 6$, which concurrently drive a 5 -bar (2DoF) mechanism, whose end-effector is used to push the box out of the folding group. Axis $\mathcal{A} 3$, which is the most prominent load, consists of a 4-bar driving an end-effector equipped with a pneumatic vacuum-cup, thus resulting in a bigger inertia. Each transmission chain is modeled by a single mechanism block with 
an overall efficiency defined according to Eq. (4), with $\eta_{\text {dir }}=\eta_{i n v}=0.9$. Other than that, the load is purely inertial.

The DC-BUS is designed such as the DC tension matches the AC peak. A grid alternate tension of $400 \mathrm{~V}$ leads to $V_{D C}=560 \mathrm{~V}$.

Table 3: Load characteristics for the packaging machine servo axes.

\begin{tabular}{|c|c|c|c|c|}
\hline Name & mechanism Type & $L_{R M S}[\mathrm{Nm}]$ & $\omega_{l, R M S}[\mathrm{rad} / \mathrm{s}]$ & $E_{m}[\mathrm{~J}]$ \\
\hline $\mathcal{A} 1$ & 4-bar & 1.0747 & 67.75 & 0.83 \\
\hline $\mathcal{A} 2$ & double 4-bar & 5.7297 & 70.09 & 4.93 \\
\hline $\mathcal{A} 3$ & 4-bar & 6.2316 & 55.48 & 2.97 \\
\hline $\mathcal{A} 4$ & 4-bar & 1.9041 & 52.96 & 1.13 \\
\hline $\mathcal{A} 5$ & 5-bar & 3.8885 & 35.76 & 1.56 \\
\hline $\mathcal{A} 6$ & 5-bar & 1.7614 & 39.68 & 0.38 \\
\hline $\mathcal{A} 7$ & 4-bar & 0.45676 & 60.81 & 0.28 \\
\hline $\mathcal{A} 8$ & 4-bar & 0.94313 & 63.72 & 0.73 \\
\hline
\end{tabular}

\subsubsection{Optimization with a unique motor-reducer pair}

1-stage coaxial gearbox reducers by B\&R are considered (RGP40 series, size 60, Tab. 9 [33]). The inverter modules are ACOPOSmulti by B\&R, reported in Tab. 10 Standard self-cooled PMSMs are considered from two different manufacturers: ELAU self-cooled motors [16] are reported in Tab. 6] with standard winding arrangement. B\&R motors (8SL series [17]) are chosen with windings arrangement corresponding to $\omega_{m, n}=3000 \mathrm{rpm}$ (Tab. 7). For these sets of motors, we observed that the quadratic term in the model of iron losses in negligible $\left(k_{f 2}=0\right)$; also, we set $d_{m}=0$ since no explicit damping sources are present (the self-cooled motors operate in standard atmosphere), and no experimental data are available for this application to obtain an accurate estimation of $d_{m}$. The resulting coefficients $k_{t 1}, k_{t 2}, k_{f 1}$ are reported for ELAU motors in Tab. 6 as an example; for the complete set of motor data see [32]. The fitting methods 
are the ones indicated in Tab. 2

One could choose an optimal motor-reducer pair for each servo-axis in order to minimize, for example, the energy consumption. However, this approach is not satisfactory for this specific case, since the company goal is to select the minimum number of different components employed in a single machine. This is justified by several reasons: logistic handling of components and spare parts, facilitation in mounting and maintenance, etc. Other than that, looking for the global optimum of a specific axis may not be convenient, if the energy consumption of that axis is negligible compared to others. In order to address these issues, we set up an optimization procedure with the final goal of finding a unique motor-reducer pair, able to drive all servo-axes while minimizing the global energy spent in the machine section, namely:

$$
\tilde{E}_{e l, t o t}(\alpha, \beta)=\sum_{a=1}^{8} E_{e l, t o t, a}(\alpha, \beta)
$$

where $E_{e l, t o t, a}$ is the energy for $a$-th axis $\mathcal{A}$ as defined in Eq. (27). This allows the contribution of the more demanding axes to be implicitly weighted more. The constraints in Eq. 26 must be respected at the same time for each axis, i.e. the global solution must be found in the feasibility region which is the intersection of the feasibility regions of all axes. Figure 11 shows function $\tilde{E}_{e l, t o t}(\alpha, \beta)$ in a color map for the B\&R catalog. For the sake of clarity, the results are presented with respect to rated torque $M_{n}$ rather than $\alpha$. The black continuous line marks the total feasibility zone corresponding to the motor thermal problem condition, while the green dashed line marks the total feasibility zone corresponding to the maximum torque condition. The global optimum (red diamond) is found for $M_{n}^{*}=8.22 \mathrm{Nm}$ and $\beta^{*}=1.04$, leading to $\tilde{E}_{e l, t o t}=107.4 \mathrm{~J}$.

The same analysis is carried out with ELAU motors, and results are shown in Fig. 12 The global optimum (red diamond) is at $M_{n}^{*}=7.45 \mathrm{Nm}$ and $\beta^{*}=1.26$, leading to $\tilde{E}_{e l, t o t}=112.5 \mathrm{~J}$. Even if the shape of the energy function varies, the results are consistent with those obtained with $\mathrm{B} \& \mathrm{R}$ motors in terms of trend and magnitudes. 


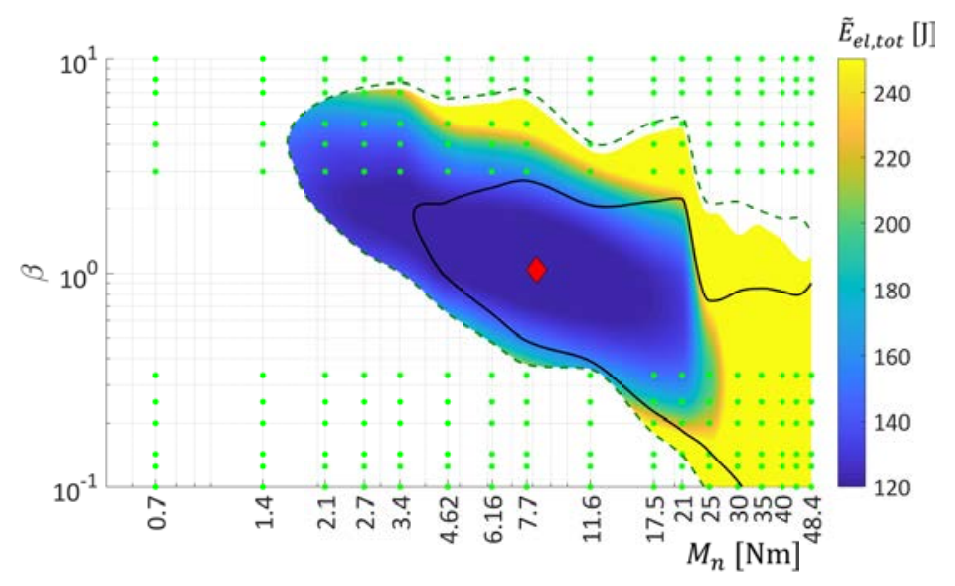

Figure 11: Optimization of the automatic packaging machine with B\&R motors and a single motor-reducer unit. The global optimum (red diamond) is at $M_{n}^{*}=8.22 \mathrm{Nm}, \beta^{*}=1.04$, and $\tilde{E}_{e l, t o t}=107.4 \mathrm{~J}$. (For interpretation of the references to colour in this figure legend, the reader is referred to the web version of this article.)

The theoretical optima for ELAU and B\&R catalogs and the information given in Figs. 11 and 12 open the way for some interesting considerations, since for this application the high-efficiency region is around the value $\beta=1$. This suggests the removal of the reducer, with a number of benefits such as: reduction of fixed costs, improvement of the reliability and stiffness of the transmission chain, lower backlash problems, etc.

Therefore, optimization is carried out for the same problem, but with the additional constraint $\beta=1$ (and thus $J_{r}=0$ and $\eta_{r}=1$ ). The problem becomes mono-dimensional, i.e. it reduces to find the value of $\alpha$ that minimizes $\tilde{E}_{e l, t o t}(\alpha)$ in Eq. (32). As for the B\&R catalog (Tab. 7) the theoretical optimum is $M_{n}^{*}=$ $8.64 \mathrm{Nm}$, with $\tilde{E}_{e l, t o t}=107.5 \mathrm{~J}$, and the closest commercial components are $\mathcal{M} 8\left(M_{n, 8}=7.7 \mathrm{Nm}\right)$ and $\mathcal{M} 9\left(M_{n, 9}=11.6 \mathrm{Nm}\right)$. In this case, the theoretical optimum $M_{n}^{*}$ is in a intermediate position between $\mathcal{M} 8$ and $\mathcal{M} 9$, which are relatively far from each other. If the application is particularly important, the user could consider the possibility to design a tailor-made motor with an intermediate size. As for ELAU (Tab. 6), the theoretical optimum is $M_{n}^{*}=7.50$ 


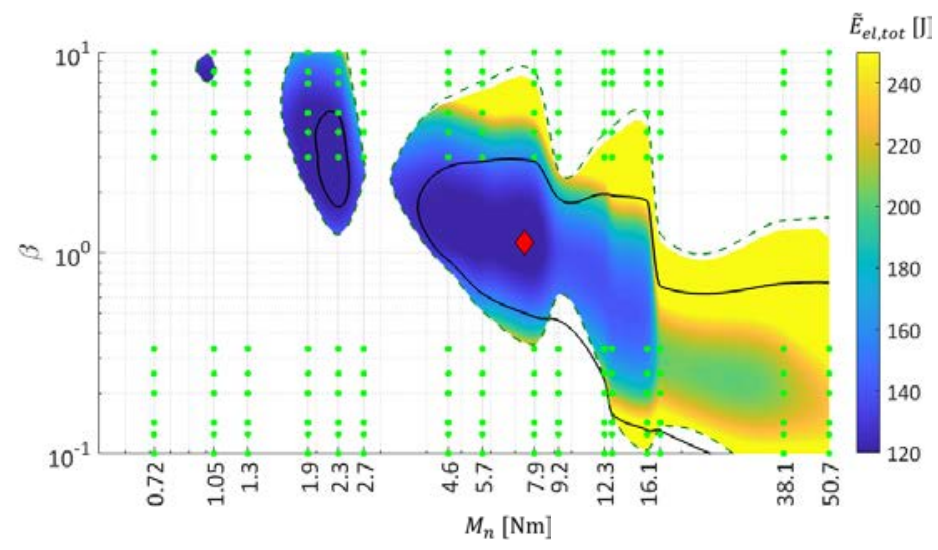

Figure 12: Optimization of the automatic packaging machine with ELAU motors and a single motor-reducer unit. The global optimum (red diamond) is at $M_{n}^{*}=7.45 \mathrm{Nm}, \beta^{*}=1.26$, and $\tilde{E}_{e l, t o t}=112.5 \mathrm{~J}$. (For interpretation of the references to colour in this figure legend, the reader is referred to the web version of this article.)

$\mathrm{Nm}$, with $\tilde{E}_{e l, t o t}=113.03 \mathrm{~J}$, leading to similar results.

The results obtained in this Section were actually used to perform the motor selection in the IMA multi-axis packaging machine, which currently employs the motor ELAU SH100-30 $\left(M_{n, 10}=7.9 \mathrm{Nm}\right)$, without reducer, for each one of the 8 axes.

\section{Conclusions}

This work dealt with the optimal selection of the motor-reducer unit in 1-DoF servo-axes composed by an inverter, a permanent-magnet synchronous motor (PMSM), a mechanical transmission, and an end-effector. Such actuators are widespread in modern servo-controlled machines, which may contain up to dozens or hundreds of servo-axes. In this scenario, the optimal selection of actuators gains a crucial importance. This work aimed at defining a novel procedure for the optimal selection of the motor and the reducer, given one or more user-defined objective functions, with a particular attention to energy efficiency. 
The first part of the work presented the electro-mechanical model of the servo-axis. The model took into account both mechanical and electrical varia-

a aeronautical applications, and a high-frequency multi-axis automatic machine for packaging tasks.

Future developments of our work will be focused on: refining the model of 
electrical losses in the inverter, thus making it independent from the availability

of experimental data; extending the portfolio of electrical models to other types of motors; introducing additional motor indices to accomodate different designs (e.g. winding arrangements); enhancing the efficiency and robustness of the optimization-solving algorithm by developing a bespoke procedure; studying to which extent the theoretical optimum found by continuous optimization is sensitive to the fitting methods used to generate the continuous catalog from a discrete one.

\section{Acknowledgments}

The work reported in this paper was partially developed within the project Sinergie, funded by the 2014-2020 Emilia-Romagna Regional Operational Programme (ROP) of the European Regional Development Fund (ERDF). The authors gratefully acknowledge the support of the companies IMA S.P.A. and L3-Calzoni. The first author would like to especially thank Dr. Yuanqing Wu for revising his $\mathrm{PhD}$ Thesis and for helping out with some of the figures.

\section{References}

[1] K. A. Pasch, W. P. Seering, On the drive system for high-performance machines, ASME Journal of Mechanisms, Transmissions, and Automation in Design 106 (1) (1984) 102-108. doi:10.1115/1.3258545.

[2] H. Van de Straete, P. Degezelle, J. D. Schutter, R. J. M. Belmans, Servo motor selection criterion for mechatronic applications, IEEE/ASME Transactions on Mechatronics 3 (1) (1998) 43-50. doi:10.1109/3516.662867

[3] F. Roos, H. Johansson, J. Wikander, Optimal selection of motor and gearhead in mechatronic applications, Mechatronics 16 (1) (2006) $63-72$. doi:http://dx.doi.org/10.1016/j.mechatronics.2005.08.001

[4] G. Cusimano, A procedure for a suitable selection of laws of motion and electric drive systems under inertial loads, Mechanism and Machine 

S0094-114X(03) 00006-5.

[5] G. Cusimano, Optimization of the choice of the system electric drivedevice transmission for mechatronic applications, Mechanism and Machine Theory 42 (1) (2007) 48 - 65. doi:http://dx.doi.org/10.1016/j. mechmachtheory.2006.02.003

[6] G. Cusimano, F. Casolo, An almost comprehensive approach for the choice of motor and transmission in mechatronics applications: Motor thermal problem, Mechatronics 40 (2016) 96-105.

815

[7] H. Giberti, S. Cinquemani, G. Legnani, Effects of transmission mechanical characteristics on the choice of a motor-reducer, Mechatronics 20 (5) (2010)

$604-610$. doi:http://dx.doi.org/10.1016/j.mechatronics.2010.06. 006.

[8] H. Giberti, S. Cinquemani, G. Legnani, A practical approach to the se820

n Design of Structures and Machines 39 (3) (2011) 303-319. arXiv:http://

1. dx.doi.org/10.1080/15397734.2011.543048 doi:10.1080/15397734. 2011.543048

[9] H. Giberti, A. Clerici, S. Cinquemani, Specific accelerating factor: One more tool in motor sizing projects, Mechatronics 24 (7) (2014) 898-905. doi:10.1016/j.mechatronics.2013.11.007

[10] G. Cusimano, Influence of the reducer efficiencies on the choice of motor and transmission: Torque peak of the motor, Mechanism and Machine Theory 67 (2013) 122-151.

${ }_{830}$ [11] S. Rezazadeh, J. W. Hurst, On the optimal selection of motors and transmissions for electromechanical and robotic systems, in: 2014 IEEE/RSJ Int. Conference on Intelligent Robots and Systems, Chicago, Illinois, USA, 2014, pp. 4605-4611. doi:10.1109/IROS.2014.6943215. 
[12] T. Verstraten, G. Mathijssen, R. Furnémont, B. Vanderborght, D. Lefeber, Modeling and design of geared dc motors for energy efficiency: Comparison between theory and experiments, Mechatronics 30 (2015) 198-213. doi: 10.1016/j.mechatronics.2015.07.004.

[13] T. Verstraten, R. Furnémont, G. Mathijssen, B. Vanderborght, D. Lefeber, Energy consumption of geared dc motors in dynamic applications: Comparing modeling approaches, IEEE Robotics and Automation Letters 1 (1) (2016) 524-530.

[14] A. Wang, S. Kim, Directional efficiency in geared transmissions: Characterization of backdrivability towards improved proprioceptive control, in: 2015 IEEE International Conference on Robotics and Automation (ICRA), 2015, pp. 1055-1062. doi:10.1109/ICRA.2015.7139307.

[15] T. Herold, D. Franck, E. Lange, K. Hameyer, Extension of a d-q model of a permanent magnet excited synchronous machine by including saturation, cross-coupling and slotting effects, in: 2011 IEEE Int. Electric Machines Drives Conference (IEMDC), Niagara Falls, Ontario, Canada, 2011, pp. 1363-1367. doi:10.1109/IEMDC.2011.5994804.

[16] Schneider Electric, PacDrive SH Servo Motor: Operating Manual (2014).

[17] B\&R, 8LS three-phase synchronous motors, Users Manual (2016).

[18] A. E. Fitzgerald, J. Kingsley, U. D., Electric Machinery. A.E. Fitzgerald, Charles Kingsley, JR., Stephen D. Umans, 6th Edition, McGraw-Hill, 2003.

[19] C. P. Steinmetz, On the law of hysteresis, Proceedings of the IEEE 72 (2) (1984) 197-221. doi:10.1109/PROC.1984.12842

[20] G. R. Slemon, X. Liu, Core losses in permanent magnet motors, IEEE 1] Transactions on Magnetics 26 (5) (1990) 1653-1655. doi:10.1109/20. 104480 
[29] PHASE Motion Control, U3 Servomotors (2017). IPEMC.2012.6258942 train, Tech report, B\&R (2013). 1-6. doi:10.1109/VPPC.2014.7007018. LXM32_DC_bus_Manual_V101_EN.pdf ing systems, Cambridge University Press, 2006. gramming 89 (1) (2000) 149-185. tion, INFORMS Journal on Computing 19 (2007) 328-340. doi:10.1137/S003614450242889.

21] W. Hassan, B. Wang, Efficiency optimization of pmsm based drive system, in: Proceedings of The 7th Int. Power Electronics and Motion Control

n Conference, Harbin, China, Vol. 2, 2012, pp. 1027-1033. doi:10.1109/

[22] Forthuberf, A. Holzleitner, A guide to calculate power losses of a drive

[23] Q. K. Nguyen, J. Roth-Stielow, Analysis and modelling of the losses for the electrical drive system of an electric vehicle, in: 2014 IEEE Vehicle Power and Propulsion Conference (VPPC), Coimbra, Portugal, 2014, pp.

[24] Schneider Electric, LXM32 - Common DC bus Application note (2009).

1 URL https://www.schneider-electric.com/resources/sites/

SCHNEIDER_ELECTRIC/content/live/FAQS/238000/FA238474/en_US/

[25] R. Baldick, Applied optimization: formulation and algorithms for engineer-

[26] R. H. Byrd, J. C. Gilbert, J. Nocedal, A trust region method based on interior point techniques for nonlinear programming, Mathematical Pro-

[27] Z. Ugray, L. Lasdon, J. C. Plummer, F. Glover, J. Kelly, R. Marti, Scatter search and local nlp solvers: A multistart framework for global optimiza-

[28] T. Kolda, R. Lewis, V. Torczon, Optimization by direct search: New perspectives on some classical and modern methods, SIAM Review 45 (3) (2003) 385-482. arXiv:https://doi.org/10.1137/S003614450242889

URL WWW . phase.eu 
[30] LENZE, L-force Gearboxes (2011).

URL www. lenze.com

890

[31] B\&R, ACOPOSmulti User's Manual (2014).

(1) URL https://www.br-automation.com/en-au/ downloads/motion-control/drive-systems/acoposmulti/ acoposmulti-users-manual/

[32] F. Meoni, M. Carricato, [dataset] motor database for the optimal selection of motor-reducer unit by continuous approach, mendeley data, v1. doi: 10.17632/tpb5tfszht. 1 .

[33] B\&R, Standard Planetary Gearboxes (2016).

a URL ww. br-automation.com/en/products/motion-control/ 8g-standard-planetary-gearbox/ 
APPENDIX 1 : Tables

Table 4: Phase motor database (forced cooling, U3 series [29]) for nominal speed $\omega_{m, n}=2000$ $\mathrm{rpm}$ and $\omega_{m, n}=3000$.

\begin{tabular}{|c|c|c|c|c|c|c|c|}
\hline \multicolumn{4}{|c|}{} & \multicolumn{2}{c|}{$\omega_{m, n}=2000 \mathrm{rpm}$} & \multicolumn{2}{c|}{$\omega_{m, n}=3000 \mathrm{rpm}$} \\
\hline Index & Name & $J_{m}\left[\mathrm{~kg} \mathrm{~cm}^{2}\right]$ & $m_{m o t}[\mathrm{~kg}]$ & $M_{n}[\mathrm{Nm}]$ & $k_{T}[\mathrm{Nm} / \mathrm{A}]$ & $M_{n}[\mathrm{Nm}]$ & $k_{T}[\mathrm{Nm} / \mathrm{A}]$ \\
\hline $\mathcal{M} 1$ & U307F20 & 14 & 19 & 28 & 2.64 & 26.5 & 1.9 \\
\hline $\mathcal{M} 2$ & U307F30 & 20 & 25 & 42.5 & 2.69 & 41.6 & 1.9 \\
\hline $\mathcal{M} 3$ & U307F40 & 26 & 31 & 57.5 & 2.53 & 55 & 1.9 \\
\hline $\mathcal{M} 4$ & U310F07 & 90 & 56 & 72 & 2.73 & 70 & 1.82 \\
\hline $\mathcal{M} 5$ & U310F10 & 130 & 70 & 97 & 2.86 & 95 & 1.82 \\
\hline $\mathcal{M} 6$ & U310F13 & 170 & 78 & 142 & 2.55 & 135 & 1.85 \\
\hline $\mathcal{M} 7$ & U313F20 & 190 & 138 & 260 & 2.44 & 240 & 1.84 \\
\hline $\mathcal{M} 8$ & U313F30 & 590 & 177 & 380 & 2.75 & 320 & 1.84 \\
\hline $\mathcal{M} 9$ & U313F40 & 780 & 211 & 480 & 2.44 & 460 & 1.84 \\
\hline
\end{tabular}


Table 5: ELAU motor database (forced cooling, SH series 16]).

\begin{tabular}{|c|c|c|c|c|c|}
\hline Index & Name & $J_{m}\left[\mathrm{~kg} \mathrm{~cm}^{2}\right]$ & $m_{m o t}[\mathrm{~kg}]$ & $M_{n}[\mathrm{Nm}]$ & $\omega_{m, n}[\mathrm{rpm}]$ \\
\hline $\mathcal{M}_{1}$ & SH-10050030 & 1.4 & 4.3 & 3.5 & 5000 \\
\hline $\mathcal{M}_{2}$ & SH-10040060 & 2.31 & 5.8 & 6.4 & 4000 \\
\hline $\mathcal{M}_{3}$ & SH-10040080 & 3.22 & 7.5 & 9.0 & 4000 \\
\hline $\mathcal{M}_{4}$ & SH-10030100 & 4.22 & 9.2 & 12.8 & 3000 \\
\hline $\mathcal{M}_{5}$ & SH-14030120 & 7.41 & 11.9 & 13.3 & 3000 \\
\hline $\mathcal{M}_{6}$ & SH-14030200 & 12.68 & 16.6 & 25 & 3000 \\
\hline $\mathcal{M}_{7}$ & SH-14030270 & 17.94 & 21.3 & 33 & 3000 \\
\hline $\mathcal{M}_{8}$ & SH-14030330 & 23.7 & 26 & 35.2 & 3000 \\
\hline $\mathcal{M}_{9}$ & SH-20520650 & 129 & 50 & 56.8 & 2000 \\
\hline $\mathcal{M}_{10}$ & SH-20520900 & 190 & 67 & 71.9 & 2000 \\
\hline
\end{tabular}


Table 6: ELAU Motor database (self-cooled, SH series [16]).

\begin{tabular}{|c|c|c|c|c|c|c|c|}
\hline Index & Name & $M_{n}[\mathrm{Nm}]$ & $\omega_{m, n}[\mathrm{rpm}]$ & $J_{m}\left[\mathrm{~kg} \mathrm{~cm}^{2}\right]$ & $k_{t 1}[\mathrm{Nm} / \mathrm{A}]$ & $k_{t 2}\left[\mathrm{Nm} / \mathrm{A}^{2}\right]$ & $k_{f 1}[\mathrm{~W} / \mathrm{rpm}]$ \\
\hline $\mathcal{M} 1$ & SH-05580005 & 0.48 & 8000 & 0.059 & 0.787 & $-93.0 \cdot 10^{-3}$ & $0.3 \cdot 10^{-3}$ \\
\hline $\mathcal{M} 2$ & SH-05580009 & 0.72 & 8000 & 0.096 & 0.705 & $-38.5 \cdot 10^{-3}$ & $0.3 \cdot 10^{-3}$ \\
\hline $\mathcal{M} 3$ & SH-05580013 & 1.05 & 8000 & 0.134 & 0.797 & $-39.8 \cdot 10^{-3}$ & $0.8 \cdot 10^{-3}$ \\
\hline $\mathcal{M} 4$ & SH-07060010 & 1.3 & 6000 & 0.25 & 0.870 & $-45.0 \cdot 10^{-3}$ & $1.3 \cdot 10^{-3}$ \\
\hline $\mathcal{M} 5$ & SH-07060020 & 1.9 & 6000 & 0.41 & 0.777 & $-11.3 \cdot 10^{-3}$ & $1.2 \cdot 10^{-3}$ \\
\hline $\mathcal{M} 6$ & SH-07060030 & 2.3 & 6000 & 0.58 & 0.786 & $-7.2 \cdot 10^{-3}$ & $2.4 \cdot 10^{-3}$ \\
\hline $\mathcal{M} 7$ & SH-10050030 & 2.7 & 5000 & 1.4 & 1.007 & $-17.3 \cdot 10^{-3}$ & $5.2 \cdot 10^{-3}$ \\
\hline $\mathcal{M} 8$ & SH-10040060 & 4.6 & 4000 & 2.31 & 1.257 & $-10.9 \cdot 10^{-3}$ & $4.8 \cdot 10^{-3}$ \\
\hline $\mathcal{M} 9$ & SH-10040080 & 5.7 & 4000 & 3.22 & 1.245 & $-8.7 \cdot 10^{-3}$ & $8.0 \cdot 10^{-3}$ \\
\hline $\mathcal{M} 10$ & SH-10030100 & 7.9 & 3000 & 4.22 & 1.627 & $-1.1 \cdot 10^{-3}$ & $12.3 \cdot 10^{-3}$ \\
\hline $\mathcal{M} 11$ & SH-14030120 & 9.2 & 3000 & 7.41 & 1.442 & $-6.9 \cdot 10^{-3}$ & $14.6 \cdot 10^{-3}$ \\
\hline $\mathcal{M} 12$ & SH-14030200 & 12.3 & 3000 & 12.68 & 1.474 & $-2.5 \cdot 10^{-3}$ & $33.5 \cdot 10^{-3}$ \\
\hline $\mathcal{M} 13$ & SH-14030270 & 12.9 & 3000 & 17.94 & 1.584 & $-1.7 \cdot 10^{-3}$ & $50.5 \cdot 10^{-3}$ \\
\hline $\mathcal{M} 14$ & SH-14030330 & 16.1 & 3000 & 23.7 & 1.584 & $-2.1 \cdot 10^{-3}$ & $51.2 \cdot 10^{-3}$ \\
\hline $\mathcal{M} 15$ & SH-20530360 & 17.5 & 3000 & 71.4 & 1.815 & $-6.3 \cdot 10^{-3}$ & $57.8 \cdot 10^{-3}$ \\
\hline $\mathcal{M} 16$ & SH-20520650 & 38.1 & 2000 & 129 & 2.423 & $-1.6 \cdot 10^{-3}$ & $110.8 \cdot 10^{-3}$ \\
\hline $\mathcal{M} 17$ & SH-20520900 & 50.7 & 2000 & 190 & 2.822 & $-2.91 \cdot 10^{-3}$ & $130.2 \cdot 10^{-3}$ \\
\hline
\end{tabular}


Table 7: B\&R motor database (self-cooled, 8LSA series [17]) for nominal speed $\omega_{m, n}=3000$ rpm.

\begin{tabular}{|c|c|c|c|}
\hline Index & Name & $M_{n}[\mathrm{Nm}]$ & $J_{m}\left[\mathrm{~kg} \mathrm{~cm}^{2}\right]$ \\
\hline $\mathcal{M} 1$ & 8LSA33-xx030 & 0.7 & 0.4 \\
\hline $\mathcal{M} 2$ & 8LSA34-xx030 & 1.4 & 0.65 \\
\hline $\mathcal{M} 3$ & 8LSA35-xx030 & 2.1 & 0.9 \\
\hline $\mathcal{M} 4$ & 8LSA36-xx030 & 2.7 & 1.15 \\
\hline $\mathcal{M} 5$ & 8LSA37-xx030 & 3.4 & 1.38 \\
\hline $\mathcal{M} 6$ & 8LSA44-xx030 & 4.62 & 2.73 \\
\hline $\mathcal{M 7}$ & 8LSA45-xx030 & 6.16 & 3.58 \\
\hline $\mathcal{M} 8$ & 8LSA46-xx030 & 7.7 & 4.39 \\
\hline $\mathcal{M} 9$ & 8LSA55-xx030 & 11.6 & 8.19 \\
\hline $\mathcal{M} 10$ & 8LSA64-xx030 & 17.5 & 13.1 \\
\hline $\mathcal{M} 11$ & 8LSA65-xx030 & 21 & 15.6 \\
\hline $\mathcal{M} 12$ & 8LSA74-xx030 & 25 & 60 \\
\hline $\mathcal{M} 13$ & 8LSA75-xx030 & 30 & 74 \\
\hline $\mathcal{M} 14$ & 8LSA76-xx030 & 35 & 102 \\
\hline $\mathcal{M} 15$ & 8LSA77-xx030 & 40 & 130 \\
\hline $\mathcal{M} 16$ & 8LSA78-xx030 & 44 & 158 \\
\hline $\mathcal{M} 17$ & 8LSA84-xx030 & 48.4 & 114 \\
\hline
\end{tabular}


Table 8: Lenze reducer database: 2-stage parallel axes gearboxes (GFL6 series [30), efficiency $\eta_{r}=0.96$.

\begin{tabular}{|c|c|c|}
\hline Index & $\beta_{k}$ & $J_{r}\left[\mathrm{~kg} \mathrm{~cm}^{2}\right]$ \\
\hline $\mathcal{R}_{1}$ & 3.675 & 7.755 \\
\hline $\mathcal{R}_{2}$ & 5.211 & 6.636 \\
\hline $\mathcal{R}_{3}$ & 5.750 & 6.044 \\
\hline $\mathcal{R}_{4}$ & 6.450 & 3.651 \\
\hline $\mathcal{R}_{5}$ & 7.147 & 4.044 \\
\hline $\mathcal{R}_{6}$ & 8.400 & 4.264 \\
\hline $\mathcal{R}_{7}$ & 9.463 & 3.879 \\
\hline $\mathcal{R}_{8}$ & 10.092 & 2.520 \\
\hline $\mathcal{R}_{9}$ & 11.520 & 1.730 \\
\hline $\mathcal{R}_{10}$ & 12.978 & 2.610 \\
\hline $\mathcal{R}_{11}$ & 14.743 & 1.950 \\
\hline $\mathcal{R}_{12}$ & 16.128 & 1.680 \\
\hline $\mathcal{R}_{13}$ & 18.169 & 1.570 \\
\hline $\mathcal{R}_{14}$ & 20.571 & 1.190 \\
\hline $\mathcal{R}_{15}$ & 23.175 & 1.130 \\
\hline $\mathcal{R}_{16}$ & 25.200 & 0.904 \\
\hline $\mathcal{R}_{17}$ & 28.389 & 0.861 \\
\hline $\mathcal{R}_{18}$ & 32.800 & 0.581 \\
\hline $\mathcal{R}_{19}$ & 36.951 & 0.556 \\
\hline $\mathcal{R}_{20}$ & 40.800 & 0.425 \\
\hline $\mathcal{R}_{21}$ & 45.963 & 0.407 \\
\hline $\mathcal{R}_{22}$ & 52.800 & 0.264 \\
\hline $\mathcal{R}_{23}$ & 59.481 & 0.251 \\
\hline $\mathcal{R}_{24}$ & 64.080 & 0.193 \\
\hline $\mathcal{R}_{25}$ & 72.189 & 0.187 \\
\hline $\mathcal{R}_{26}$ & 81.000 & 0.125 \\
\hline $\mathcal{R}_{27}$ & 91.250 & 0.121 \\
\hline
\end{tabular}


Table 9: B\&R reducer database: 1-stage planetary gearboxes (8GP series [33]), efficiency $\eta_{r}=0.96$.

\begin{tabular}{|c|c|c|c|}
\hline Index & Name & $\beta_{k}$ & $J_{r}\left[\mathrm{~kg} \mathrm{~cm}^{2}\right]$ \\
\hline $\mathcal{R}_{1}$ & 8GP40-060hh010 (flipped) & $1 / 10$ & 6.400 \\
\hline $\mathcal{R}_{2}$ & 8GP40-060hh008 (flipped) & $1 / 8$ & 4.160 \\
\hline $\mathcal{R}_{3}$ & 8GP40-060hh007 (flipped) & $1 / 7$ & 3.528 \\
\hline $\mathcal{R}_{4}$ & 8GP40-060hh005 (flipped) & $1 / 5$ & 1.950 \\
\hline $\mathcal{R}_{5}$ & 8GP40-060hh004 (flipped) & $1 / 4$ & 1.488 \\
\hline $\mathcal{R}_{6}$ & 8GP40-060hh003 (flipped) & $1 / 3$ & 1.215 \\
\hline $\mathcal{R}_{7}$ & $8 \mathrm{GP} 40-060 \mathrm{hh} 003$ & 3 & 0.135 \\
\hline $\mathcal{R}_{8}$ & $8 \mathrm{GP} 40-060 \mathrm{hh} 004$ & 4 & 0.093 \\
\hline $\mathcal{R}_{9}$ & 8GP40-060hh005 & 5 & 0.078 \\
\hline $\mathcal{R}_{10}$ & 8GP40-060hh007 & 7 & 0.072 \\
\hline $\mathcal{R}_{11}$ & 8GP40-060hh008 & 8 & 0.065 \\
\hline $\mathcal{R}_{12}$ & 8GP40-060hh010 & 10 & 0.064 \\
\hline
\end{tabular}


Table 10: B\&R inverter database (ACOPOSmulti series [31]) for switching frequency $f_{s}=5$ $\mathrm{kHz}$ and $f_{s}=10 \mathrm{kHz}$.

\begin{tabular}{|c|c|c|c|c|c|c|c|c|}
\hline Name & \multicolumn{2}{|c|}{$f_{s}=5 \mathrm{kHz}$} & \multicolumn{3}{c|}{$f_{s}=10 \mathrm{kHz}$} & \multicolumn{2}{c|}{} \\
\hline Name & $k_{i 2}$ & $k_{i 1}$ & $k_{i 0}$ & $k_{i 2}$ & $k_{i 1}$ & $k_{i 0}$ & $I_{n}[\mathrm{~A}]$ & $I_{\text {max }}[\mathrm{A}]$ \\
\hline 8BVI0014HxS & 0.60 & 1.30 & 60 & 0.97 & 0.50 & 110 & 1.9 & 4.7 \\
\hline 8BVI0028HxS & 0.60 & 1.30 & 60 & 0.97 & 0.50 & 110 & 3.8 & 9.5 \\
\hline 8BVI0055HxS & 0.60 & 1.30 & 60 & 0.97 & 0.50 & 110 & 7.6 & 18.9 \\
\hline 8BVI0110HxS & 0.15 & 5.60 & 55 & 0.49 & 4.70 & 95 & 15.1 & 37.7 \\
\hline 8BVI0220HxS & 0.13 & 5.50 & 40 & 0.43 & 3.70 & 110 & 22 & 54.9 \\
\hline 8BVI0330HxS & 0.07 & 7.30 & 40 & 0.20 & 11.1 & 130 & 33 & 83 \\
\hline 8BVI0440HxS & 0.07 & 7.30 & 40 & 0.20 & 11.1 & 130 & 44 & 88 \\
\hline 8BVI0660HxS & 0.03 & 7.90 & 90 & 0.11 & 11.0 & 185 & 66 & 132 \\
\hline 8BVI0880HxS & 0.03 & 7.90 & 90 & 0.11 & 11.0 & 185 & 88 & 176 \\
\hline 8BVI1650HxS & 0.001 & 9.9 & 165 & 0.17 & 10.8 & 320 & 165 & 330 \\
\hline
\end{tabular}

